\title{
Arbeitspolitik in digitalen Zeiten: Chancen und Herausforderungen für Arbeitsbeziehungen, Arbeitsgestaltung und Organisation
}

\author{
Thomas Haipeter, Fabian Hoose, Sophie Rosenbohm
}

\section{Digitalisierung der Arbeitswelt}

Digitalisierung gilt seit einigen Jahren als Megatrend von Wirtschaft und Arbeit. Der Digitalisierung werden nicht weniger als revolutionäre und radikale Auswirkungen auf die bestehenden Strukturen von Wirtschaftssektoren, Beschäftigung, Qualifikationsbedarfen oder Arbeitszeiten zugesprochen. Im Zentrum der damit verbundenen Visionen und Leitbilder steht häufig der Gedanke der Vernetzung von physischer (oder digitaler) Produktion über das Internet, welche neuartige Möglichkeiten der Verbindung und Koordinierung physischer und informationeller Prozesse - Maschinen, Güter, Menschen und Informationen - bereithält und damit Echtzeitsteuerung, die Nutzung algorithmische Verfahren und die Verfügbarmachung von Big Data verspricht. Postuliert wird dabei eine neue Qualität dieser Veränderungsprozesse, die sich damit von bisherigen Informatisierungsprozessen deutlich unterscheiden. Diese Zusammenhänge werden in Deutschland vor allem unter der Überschrift Industrie 4.0 diskutiert. Darüber hinaus gibt es mit Blick auf die Entwicklung von Arbeit aber auch weitere Schwerpunkte der Aufmerksamkeit, vor allem die Plattformökonomie sowie Digitalisierungsprozesse im Dienstleistungsbereich oder der Verwaltung.

Zwar wird in diesen Zusammenhang kritisch angemerkt, dass es sich bei den Diskursen um Digitalisierung nicht zuletzt um einen durch Agenda-Setting hervorgerufenen Hype handelt und von Akteuren wie Verbänden, Manager*innen, Berater*innen und Wissenschaftler*innen, jeweils mit durchaus eigenen wirtschaftlichen oder politischen Interessen, erfolgreich inszeniert wurde. Die aktuelle Reichweite der Durchdringung der Unternehmen mit digitalen Technologien - vor allem in Hinblick auf selbstständig über das Internet kommunizierender Güter und Maschinen oder dem Einsatz smarter Arbeitsmittel - liegt demnach noch weit hinter den kursierenden Leitbildern und Visionen zurück (Howaldt, Kopp und Schultze 2018; Pfeiffer 2015; Urban 2016). Aber ist daraus zu schließen, 
dass die Digitalisierung nur eine Vision neben anderen ist, die möglicherweise schnell wieder verschwindet, wenn die Kluft zwischen Konzept und Wirklichkeit offensichtlich wird? Oder ist sie nicht vielmehr eine soziale Konstruktion, die im Sinne des Thomas-Theorems die soziale Wirklichkeit prägen wird, weil sie als Leitbild das Handeln der sozialen Akteure beeinflusst und tatsächlich entsprechende Veränderungen erzeugt?

Für die zweite Annahme spricht einiges. Digitalisierung, insbesondere in der Variante der Industrie 4.0, gilt als neuer Markenkern der Wettbewerbsfähigkeit der deutschen Exportindustrie (Meyer 2020). Auf der politischen Ebene werden immer neue Kommissionen oder Ministerien eingerichtet, die sich mit dem Thema befassen und die neue politische Initiativen zur digitalen Durchdringung von Wirtschaft und Gesellschaft zu entwickeln oder die Folgewirkungen für Arbeit und Beschäftigung oder auch Datenschutz einzuschätzen und zu bearbeiten versuchen. In Innovationsprogrammen wie der aktuellen „High-Tech Strategie 2025“ der Bundesregierung werden erhebliche Finanzmittel eingesetzt, um die Entwicklung und Verbreitung der Digitalisierung in den Unternehmen und darüber hinaus zu fördern. Arbeitgeber- und Wirtschaftsverbände propagieren die wichtige Bedeutung der Digitalisierung für die wirtschaftliche Entwicklung und unterstützen Unternehmen durch Informationen und Beratung. Unternehmen erhalten auf diese Weise Anreize, auf den Zug der Digitalisierung aufzuspringen, um ihre Konkurrenzfähigkeit zu verbessern oder um ihre Fassade von Fortschrittlichkeit und Rationalität aufrechtzuerhalten. Und auch Gewerkschaften und Betriebsräte greifen das Thema auf, um Gestaltungsspielräume der Arbeit zu nutzen, die sich mit der Anwendung digitaler Technologien in den Unternehmen ergeben. Im Zusammenspiel dieser Entwicklungen gewinnt die Digitalisierung eine Eigendynamik als Gegenstand des Handelns der sozialen Akteure, die konkrete Auswirkungen darauf haben dürfte, wie Betriebe und Arbeit organisiert werden, wie sich Tätigkeiten und Qualifikationsanforderungen verändern, welche Tätigkeiten von wem in welchen Beschäftigungsformen und Branchen ausgeübt werden und schließlich, welche Tätigkeiten digitalisiert oder automatisiert werden.

Nicht zuletzt wegen dieser Veränderungen ist die Digitalisierung auch zu einem Leitthema der Sozialwissenschaften geworden, vor allem mit Blick auf die Arbeitsforschung, aber auch darüber hinaus. Inzwischen wird sogar eine digitale Soziologie zu begründen versucht mit dem Argument, dass die Digitalisierung nicht nur ein zentraler Forschungsgegenstand ist, sondern auch ein neuartiges Forschungsinstrument, weil sie neue Möglichkeiten der Produktion, Analyse und Manipulation von Daten bereithalte und damit soziales Leben und soziale Forschung auf neue Weise verbinde 
(Marres 2017; Maasen und Passoth 2020). So weit geht die Arbeitsforschung aktuell noch nicht, doch auch hier gilt, dass Themen der Digitalisierung und ihre Auswirkungen auf Arbeit und Beschäftigung zunehmend die Forschungslandschaft prägen.

Allerdings sind mit der Digitalisierung zahlreiche Fragen an die Arbeitsforschung verbunden, die alles andere als geklärt zu sein scheinen. Zumindest drei davon sollen im Folgenden näher beleuchtet werden. Dabei geht es nicht darum, die Fragen abschließend zu beantworten; zu vielen der Fragen gibt es noch keine empirisch gehaltvollen Befunde. Vielmehr sollen Problemfelder benannt werden, deren nähere Klärung durch weitere Forschung wichtig wäre und zu der auch die in diesem Band versammelten Aufsätze beitragen können.

Die erste Frage bezieht sich auf die Digitalisierung als Triebkraft für den Wandel von Arbeit, Arbeitsbedingungen und Arbeitsregulierung. Der Digitalisierung als Megatrend wird ein disruptives Potenzial unterstellt, das Tätigkeiten, Arbeitsorganisation, Qualifikationen oder Beschäftigungsstrukturen grundlegend verändern könnte. Doch was ist unter Digitalisierung eigentlich zu verstehen? Was macht eine digitale oder digitalisierte Arbeit aus? Und was lässt sich derzeit darüber sagen, wie sich die Digitalisierung auf die Entwicklung von Arbeit auswirkt?

Damit eng verbunden ist die zweite Frage, wie sich das Neue der Digitalisierung von „alten“ Herausforderungen und Rahmenbedingungen der Arbeit abhebt; zu denken ist hier an Entwicklungen wie die Globalisierung der Produktion oder die Finanzialisierung der Corporate Governance und die jeweils damit verbundenen betrieblichen oder überbetrieblichen Restrukturierungs- und Rationalisierungsstrategien, die wohl nicht einfach deshalb beendet werden, weil nun die Digitalisierung eingesetzt hat. Doch die Digitalisierung gilt häufig nicht nur als neues technologisches Paradigma, sondern auch als neuer Treiber der Akkumulation und Regulation und damit als neuer Typus des Kapitalismus. Was heißt das konkret? Und was ist mit anderen Herausforderungen? Überstrahlt (und determiniert) die technologische Entwicklung die anderen Felder institutionellen Wandels und sozialer Praktiken? Oder haben die alten Herausforderungen weiterhin Relevanz? Und wirken diese Entwicklungen in gemeinsame oder gegenläufige Richtungen?

Schließlich stellt sich drittens die Frage nach den arbeitspolitischen Herausforderungen der Digitalisierung. Gegenwärtig werden viele mögliche Entwicklungsszenarien von Arbeit unter den Vorzeichen der Digitalisierung diskutiert. Diese Vielfalt oder Offenheit lässt sich damit erklären, dass weder der technologische Wandel noch institutionelle Verschiebungen oder Unternehmensstrategien unvermittelt auf die Gestaltung, die Organi- 
sation und die Bedingungen von Arbeit wirken, sondern dass sie Gegenstand arbeitspolitischer Auseinandersetzungen und Kompromissbildungen am Arbeitsplatz, im Betrieb und im Unternehmen, aber auch auf überbetrieblicher Ebene - sei es auf nationaler Ebene, auf Branchenebene oder in Wertschöpfungsketten - sind. Damit wiederum hängen sie ab von der Durchsetzungsstärke, der Strategiebildung und den Aushandlungen der arbeitspolitischen Akteure. Welche neuen arbeitspolitischen Herausforderungen gehen mit der Digitalisierung einher? Wie verhalten sie sich zu anderen Herausforderungen? Und welche Ansatzpunkte für Strategiebildungen und neue Initiativen der Arbeitspolitik zeichnen sich ab?

\section{Digitalisierung, digitalisierte und digitale Arbeit}

Was macht die Digitalisierung der Arbeitswelt aus? Die Konturen dieses „zweiten Maschinezeitalters" werden nach Brynjolfsson und McAfee (2014) durch den dynamischen Anstieg der Leistungsfähigkeit von Hardware in puncto Speicherfähigkeiten und Geschwindigkeit sowie der digitalen Vernetzung von Informationen bestimmt. Digitale Informationstechnologien werden demnach immer mehr zu Basistechnologien von Innovationen, und sie werden immer billiger, massenhaft verfügbar und breit einsetzbar. Es sind demzufolge vor allem drei technologische Neuerungen, die den Einsatz digitaler Technologien in Unternehmen prägen: (1) die Erfassung, Vernetzung und damit Produktion digitaler Informationen und Daten im Sinne von Big Data, (2) die informationstechnologische Verbindung von Maschinen und Menschen, die diese nutzen, durch Software und Datennetze und (3) der Einsatz neuer Generationen von Maschinen und Robotern, die mit Hilfe neuer Formen der Sensorik neue Funktionen ausüben und Hand in Hand mit Menschen interagieren können. Aus dieser Entwicklung ziehen die Autoren den pessimistischen Schluss, dass alle Tätigkeiten, die auf standardisierten und routinierten Abläufen und ihrer Wiedererkennung beruhen, bald durch digitale Technologie automatisiert werden können; nur Tätigkeiten mit Innovationsgehalt, komplizierter Mustererkennung und komplexer Kommunikation werden demnach weiterhin durch menschliche Arbeit geprägt sein. Dieser Befund hat wesentlich zur Debatte um die negativen Beschäftigungseffekte der digitalen Technologien beigetragen, die Frey und Osborne mit Blick auf die USA auf $47 \%$ aller Jobs beziffert haben (Frey und Osborne 2013, S. 41). Inzwischen haben zahlreiche Studien versucht, die Beschäftigungseffekte von Digitalisierung und dadurch ermöglichter Automatisierung auch im internationalen Vergleich (u.a. Arntz, Gregory und Zierahn 2016; Degryse 
2016; OECD 2019) oder speziell für Deutschland (u.a. Bonin, Gregory und Zierahn 2015; Dengler und Matthes 2019; Südekum 2018) zu umreißen. Eine Grundannahme ist all diesen Studien gemein: dass immer mehr und immer komplexere Tätigkeiten durch den Einsatz digitaler Technik von Maschinen potenziell übernommen werden können. Digitalisierung wird als Prozess verstanden, durch den (mindestens) einzelne Tätigkeiten automatisiert werden können und dann keine menschliche Arbeitskraft mehr benötigt wird. Aufgrund der Unterschiedlichkeit sowohl der verwendeten Methoden, der betrachteten Branchen und Berufe sowie der jeweils genutzten Datenbasis fallen die Einschätzungen des jeweiligen Substituierungspotentials der Digitalisierung jedoch sehr verschieden aus (Hoose 2018, S. 19-21).

Die Unterschiede in den Prognosen sind nicht zuletzt auch darauf zurückzuführen, dass die neuen digitalen Technologien sehr vielfältige, mal einfachere, mal äußerst komplexe, Verwendungsmöglichkeiten eröffnen. Digitale Kommunikationsmedien beschleunigen die Arbeitswelt, indem der Informations- und Datenaustausch auch ortsübergreifend in Echtzeit erfolgen kann. Sie erlauben damit neue Formen des Zusammenarbeitens über die Grenzen von Betrieben hinweg und ermöglichen sowohl zeitgleiches wie auch zeitversetztes Arbeiten in neuen organisatorischen Zusammenhängen.

In den Dienstleistungsbereichen betrifft dies beispielsweise die Internetund IT-Branchen, in denen mit digitalen Instrumenten in digitalen Informationsräumen digitale Innovationen und Produkte der Hard- und Software erzeugt werden; nicht von ungefähr ist hier auch die Heimat der agilen Methoden mit kurzzyklischer Erfolgskontrolle, die die Innovationsdynamik hochhalten sollen (Boes, Kämpf, Langes und Lühr 2018). Ebenfalls findet sich bereits seit Ende der 1990er Jahre in Branchen wie dem Finanzwesen, der Musikindustrie oder dem Pressebereich ein weitreichender Einsatz digitaler Informations- und Kommunikationstechnologien mit gravierenden Folgen für Geschäftsmodelle, Wertschöpfungsketten und damit auch für Arbeit und Beschäftigung (Hirsch-Kreinsen 2018a, S. 14). Dynamisch zeigt sich der Einsatz digitaler Technologien im Versicherungs- und Bankgewerbe (Tullius 2020); hier reicht er von der Digitalisierung der Finanztransaktionen über digitale Software des Portfoliomanagements hin $\mathrm{zu}$ digitalisierten Formen der Kundenbetreuung und automatisierten Dienstleistungen und zu radikalen Innovationen wie der Block-ChainTechnologie, die als Grundlage für Kryptowährungen, also Formen digitalen Geldes, dient (Alt und Puschmann 2016; Staab und Prediger 2019, S. 153-176). 
Intensiver als diese Entwicklungen haben sich aber zwei andere Ansatzpunkte der Digitalisierung als Fokus der Arbeitsforschung etabliert: die Industrie 4.0 und die Plattformökonomie. Im industriellen Exportsektor wird die Digitalisierung unter dem Topos der „Industrie 4.0“ als vierte industrielle Revolution propagiert (acatech 2013; Spath 2013). Technologischer Kern der Industrie 4.0 ist der Einsatz digitaler Produktionstechnologien. Im Zentrum stehen dabei sogenannte Cyber-Physische Systeme (Ittermann und Niehaus 2018, S.35), mit denen Menschen, Maschinen, Materialen und physisch vorhandene Produkte mit immateriell bzw. digital vorhandenen Informationen mittels Sensorik verbunden werden und über das Internet kommunizieren können. In der Industrie 4.0 wird die Erfassung und Verknüpfung aller - sowohl der produktionsbezogenen als auch der betriebswirtschaftlichen - Daten mit einer dezentralen Selbststeuerung durch prozessbezogene Kommunikation in Echtzeit verknüpft. Auf diese Weise, so das damit verbundene Versprechen, wird nicht nur die Steuerung und Organisation der betrieblichen und überbetrieblichen Prozesse entlang der Wertschöpfungskette verbessert, sondern auch Kleinserienoder Einzelfertigung automatisierbar und betriebswirtschaftlich rentabel. Im Rahmen der Industrie 4.0 wird zudem auch der Einsatz neuer Formen der Robotik (Gerst 2016) oder IT-basierter Assistenzsysteme (Niehaus 2017; Kuhlmann 2018) diskutiert, bei denen nach ihren Potenzialen für Unterstützung und Autonomie auf der einen oder Kontrolle und Standardisierung von Arbeit auf der anderen Seite gefragt wird. Vielfach werden in derartigen digitalen Automatisierungsmöglichkeiten der Produktion ökonomisch vorteilhafte Rationalisierungspotentiale gesehen. Insofern können hinsichtlich der Industrie 4.0 „Analogien zu früheren Technikdebatten“ (Minssen 2017, S. 121), wie der Vision von gänzlich automatisierten, menschenlosen Fabriken im Rahmen des Computer Integrated Manufacturing (CIM), gezogen werden, bei denen sich technikzentrierte Hoffnungen auf etwaige Produktivitätssteigerungen letztlich nicht erfüllten.

Seit Lancierung des Konzepts wird daher zu ermitteln versucht, welche Verbreitung die Industrie 4.0 tatsächlich findet. Befragungen zeigen, dass sich die Anwendung des neuen technologischen Paradigmas auf größere Unternehmen konzentriert und dass es dabei zunächst um einzelne technologische Neuerungen geht, die an die bestehenden Produktions- und ITTechnologien andocken (Lerch, Jäger und Spomenca 2017). Für einen radikalen Bruch gibt es demnach aktuell kaum Hinweise. Hirsch-Kreinsen (2018a) unterscheidet auf der Grundlage der bisherigen Befunde mit Blick auf die Industrie 4.0 drei Typen von Unternehmen: Erstens die Vorreiterunternehmen, die digitale Technologien wie IT-gestützte Produktionsund Steuerungssysteme anwenden, Systeme vernetzen und selbststeuernde 
Systeme wie Leichtbauroboter in der Produktion einsetzen; dabei handelt es sich zumeist um Großunternehmen mit komplexen Prozessen und hohen Seriengrößen. Zweitens die abwartenden Unternehmen oder Follower, die sich an den Vorreiterunternehmen orientieren und ebenfalls an der Vernetzung der Systeme arbeiten oder auch mobile Endgeräte einführen. Drittens schließlich die Nachzügler, die nur vereinzelt digitale Steuerungssysteme aufweisen, traditionell wenig forschungs- und technologieintensiv sind und nur geringe Losgrößen produzieren. Während die erste Gruppe mit rund $20 \%$ der Unternehmen taxiert werden kann, entfallen nach Hirsch-Kreinsen (2018b, S. 9-10) auf die beiden anderen Gruppen jeweils etwa $50 \%$ und $30 \%$ der Unternehmen.

Während mit dem Fokus auf Industrie 4.0 insbesondere der Blick auf digitalisierungsbedingte Veränderungen bestehender Erwerbsarbeit, also digitalisierte Arbeit (zur Unterscheidung der Begriffe siehe Hoose 2018, S. 4), gerichtet wird, verhält sich dies bei der Plattformökonomie anders, da deren Entstehung eindeutig digital getrieben ist. Hier haben sich neue Geschäftsmodelle von Online-Plattformen etabliert, die als Intermediäre zwischen Anbieter*innen von Leistungen oder Aufträgen und Kund*innen oder Auftragnehmer*innen auftreten. Die Leistung der Plattformen als Portale besteht nicht nur darin, Anbieter- und Nachfrageseite zusammenzubringen; sie offerieren zudem weitere Dienstleistungen. Sie zergliedern Arbeitsaufträge in standardisierte Aufgaben, sie kontrollieren die Leistungserbringung über die Erfassung von Leistungsdaten, und sie nutzen dafür Rating- und Rankingsysteme (Dolata 2020, S. 193). Gegenüber anderen Plattformunternehmen, welche diverse Güter (Handelsplattformen wie Amazon), Geld (sog. Crowdfunding-Plattformen wie Kickstarter) oder Informationen (Suchmaschinen wie Google) anbieten, wird auf „Arbeitsplattformen“ (Schmidt 2016, S. 6) Arbeit für bezahlte Dienstleistungen vermittelt. Als relevant für die Arbeitsforschung hat sich die Unterscheidung vor allem von zwei Plattformtypen erwiesen: Einerseits Plattformen, worüber lokal auszuübende Tätigkeiten (,Gigs') wie Lieferer- oder Fahrdienste sowie haushaltsbezogene Dienstleistungen oder Handwerksarbeiten vermittelt werden. Davon können andererseits Crowdwork-Plattformen unterschieden werden, bei denen die Ausschreibung von digitalen Arbeitsaufträgen an nicht spezifisch bestimmte Personen, die Crowd, erfolgt. Die Gig-Economy kann vor allem als neuer Vertriebsweg für lokale Dienstleistungen betrachtet werden, bei dem zugleich digitale Technologien zur Verteilung und Kontrolle der Arbeitsleistung zum Einsatz kommen. Demgegenüber ist das Crowdworking eine neue Form des Sourcings: Auftraggeber lassen Tätigkeiten nicht oder nicht mehr in der eigenen Organisation ausführen, sondern vergeben sie, aufgefächert in Einzelaufträge, an eine 
externe Crowd (Leimeister, Durward und Zogaj 2016). Vorreiter dieser Entwicklung war IBM mit der Strategie der Generation Open (Boes, Kämpf, Langes und Lühr 2015). Hier konkurrieren nicht mehr abhängig Beschäftigte eines Unternehmens miteinander, sondern Leistungsanbieter, die in der deutschen Rechtsprechung bislang als Selbstständige eingestuft werden und damit nicht unter die Arbeitsgesetzgebung fallen oder in den Sozialversicherungssystemen pflichtversichert sind (Däubler 2015; Schwemmle und Wedde 2018). Die Ermittlung von genaueren Daten zur Verbreitung von Plattformarbeit gestaltet sich schwierig; bisherige Studien weisen jeweils unterschiedliche Probleme auf (Bonin und Rinne 2017). Huws, Spencer und Joyce (2016, S. 23) beispielsweise schätzen den Anteil von Crowdworker*innen - zu denen sie auch die Gig-Worker*innen zählen - in Deutschland auf $12 \%$; Serfling (2018, S. 13) veranschlagt den Anteil von Crowdworker*innen an der Wahlbevölkerung auf 4,8\%, und Urzì Brancati, Pesole und Férnandéz-Macías (2020, S. 16) gehen von einem Anteil von 9,1\% Plattformarbeitenden an der Erwerbsbevölkerung aus.

Crowdworking gilt dabei vielfach als „Menetekel“ (Schramm und Tietgen-Simonsen 2019) des Wandels der Arbeit in der digitalisierten Ökonomie. Huws (2016) hat für dieses neue, aus ihrer Sicht zunehmend normativ wirksame Arbeitsparadigma den Begriff „Logged Labour“ entwickelt. Logged Labour zeichnet sich demnach durch drei Charakteristika aus: Arbeitsaufträge werden in standardisierte Teilkomponenten zergliedert, Arbeit wird kontinuierlich technologisch kontrolliert und überwacht, und sie erfordert die Verbindung der Beschäftigten mit Online-Plattformen. Damit werde Arbeit zugleich formalisierter und prekärer, stärker verbunden und atomisierter.

Dieses Szenario eines digitalen Taylorismus ist auch von anderen Autoren gezeichnet worden. Am Beispiel der Logistikbeschäftigten in AmazonVerteilzentren diagnostizieren Staab und Nachtwey (2016, S. 27-29) eine neue Qualität der technologiegestützten Überwachung von Arbeitsprozessen, die sich durch die Nutzung neuer Formen der digitalen Kontrolle mithilfe von Scanner-Systemen auszeichnet. Eine ähnliche Diagnose entwickeln Butollo, Ehrlich und Engel (2017) mit Blick auf die Kontraktlogistik der Automobilindustrie und die dort inzwischen typische Verbindung von Logistiktätigkeiten und standardisierten Vormontagen. Auch hier wird die Kombination aus Standardisierung der Arbeit und neuen, technologisch basierten Kontrollformen als paradigmatisch für den Wandel der Arbeit betrachtet.

Diese Analysen ziehen zwei Fragen nach sich. Die erste Frage betrifft die Verallgemeinerbarkeit der Befunde. Die hier jeweils betrachteten Beschäftigtengruppen - und die Zahl der überhaupt einbezogenen Fälle - sind 
überaus klein. Zwar sind beispielsweise die Kontraktlogistik und die Handelslogistik wachsende Bereiche; doch bilden sie nur kleine Segmente der Industrie- oder Dienstleistungsbeschäftigung, die ohnedies für ihre problematischen Arbeitsbedingungen auch mit Blick auf Lohnstandards, Tarifbindung und Interessenvertretungen seit langem und unabhängig von der Digitalisierung bekannt sind. Es bleibt völlig offen, was nun tatsächlich neu ist und vor allem, ob und inwieweit die Befunde auf die Entwicklungstendenzen in anderen Tätigkeitsegmenten beispielsweise der industriellen Fach- und Angestelltenarbeit oder der Arbeit in öffentlichen Verwaltungen und Krankenhäusern übertragbar sind. Für die bislang in Deutschland kaum verbreiteten Variante des Crowdwork als alleinige Einnahmequelle gilt dies ohnehin, zumal die Tätigkeiten dort als heterogen bekannt sind, je nachdem, um welchen Plattformtyp es sich handelt, ob also eher standardisierte oder eher spezialisierte Aufträge dort auf den Markt gebracht werden. Und die zweite Frage lautet, ob die Digitalisierung wirklich der ursächliche und alleinige Treiber von Veränderungen ist, oder ob nicht andere Entwicklungstendenzen ebenso bedeutsam sind. Auch diese Frage wird bislang kaum behandelt.

Für beide Fragen macht es Sinn, sich zunächst einmal zu vergegenwärtigen, was denn überhaupt digitale Arbeit ist, von der am ehesten angenommen kann, dass sie wesentlich durch Digitalisierung - in welcher Form auch immer - beeinflusst wird. Eine plausible Definition lautet, dass von digitaler Arbeit dann gesprochen werden kann, wenn Arbeit mit digitalen Arbeitsmitteln an digitalen Objekten erfolgt (Hoose 2018, S. 12; siehe auch Schwemmle und Wedde 2012, S. 14-16). Gemäß dieser Definition wäre die Industriearbeit in der Kontraktlogistik ebenso wenig digital wie die Arbeit in der Handelslogistik oder auch den haushaltsnahen Dienstleistungen des Gig-Working; die Arbeit der Crowdworker*innen hingegen schon, und auch die computergestützten Arbeitsprozesse von Industrieangestellten oder Angestellten in den Dienstleistungen vom Bankgewerbe bis zum öffentlichen Dienst, und dies umso mehr, je mehr administrative und damit datenverarbeitende Funktionen dominieren und je weniger Kontakte zu Kund"innen eine Rolle spielen. Es steht zu erwarten, dass vor allem die Büroarbeit von Digitalisierungsprozessen wie dem Einsatz neuer Steuerungssoftware oder der Vernetzung von Softwaresystemen betroffen ist, weil diese ihre Arbeitsmittel - die Softwareprogramme - und ihren Arbeitsgegenstand - die Art der zu bearbeitenden Daten oder auch ihre Entscheidungsspielräume, die ihnen durch die Software eingeräumt werden oder auch nicht - unmittelbar prägen.

Dies heißt freilich nicht, dass nicht-digitale Arbeit nicht auch von der Digitalisierung betroffen wäre. Wie die zitierten Beispiele aus der Logistik 
zeigen, beeinflussen digitale Technologien auch die physische Produktionsarbeit, obwohl sie keine digitale Arbeit im engeren Sinne ist. Sofern und je mehr digitale Technologie an diesen Arbeitsplätzen Einzug hält, können die dort ausgeübten Tätigkeiten als digitalisiert bezeichnet werden, ohne damit der Definition digitaler Arbeit zu entsprechen. Die offene Frage lautet allerdings, ob diese Tätigkeiten sich damit auch zwangsläufig in die Entwicklung erweiterter Standardisierung und Kontrolle einreihen. Mit Blick auf die Industriearbeit beispielsweise herrscht darüber keine Gewissheit. Im Gegenteil, hier wird von sehr unterschiedlichen Entwicklungsszenarien der Industriearbeit ausgegangen. Hirsch-Kreinsen (2018a, S. 20) vermutet Wandlungsprozesse der Arbeitsorganisation zwischen zwei Polen: Auf der einen Seite die polarisierte Organisation mit einer Polarisierung von Aufgaben, Qualifikationen und Positionen zwischen einfachen und hochstandardisierten operativen Tätigkeiten und qualifizierten dispositiven Tätigkeiten mit hohen Handlungsspielräumen; auf der anderen Seite die Schwarm-Organisation mit qualifizierten Beschäftigten in vernetzten Strukturen mit der Hauptaufgabe, Prozessprobleme durch Stör- und Sondersituationen zu bewältigen. Damit reihen sich diese Befunde zur Digitalisierung von Arbeit ein in bereits zuvor getroffene Prognosen zu heterogenen Auswirkungen technologischer Veränderungen von Arbeit (Kern und Schumann 1984).

Ähnlich komplex sind die vorläufigen Befunde auch für andere Dimensionen von Arbeit wie die Beschäftigungssicherheit, die Qualifikationsbedarfe oder die Entgrenzung von Arbeit. Mit Blick auf die Beschäftigungsentwicklung stehen sich Szenarien drastischer Beschäftigungsverluste durch digitale Automatisierung (Frey und Osborne 2013), Prognosen mäBigen Beschäftigungsrückgangs (Bonin et al. 2015), aber auch Annahmen eines Strukturwandels mit Verlusten in bestimmten und Zuwächsen von Beschäftigung in anderen Sektoren - beispielsweise durch die Erschließung neuer Geschäftsfelder - gegenüber (Zika, Helmrich, Maier, Weber und Wolter 2018). Ähnlich ist die Lage bei den Qualifikationsbedarfen. Einige Autoren, gerade auch Verfechter des Leitbilds der Industrie 4.0, prognostizieren einen generellen Anstieg des Qualifikationsbedarfs beispielsweise für kompetente Stör- und Fehlerbeseitigung (Kagermann 2014), andere Autoren wie Brynjolfsson und McAfee (2014) vermuten eine Polarisierung und dritte gehen von zumindest aktuell geringen Veränderungen der fachlichen Qualifikationsanforderungen zumindest mit Blick auf industrielle Facharbeit aus (Abel 2018). Auch bei der Entgrenzung schließlich ist die Befundlage alles andere als klar. So bringen demnach globale IT-gestützte Informationsräume Entgrenzungspotenziale mit sich, weil sie Kommunikation zu allen Tages- und Nachtzeiten ermöglichen (und wo- 
möglich einfordern), und auch die Aufhebung der räumlichen Trennung von Arbeit und Privatleben durch Telearbeit oder mobile Arbeit kann solche Entgrenzungen befördern (Schwemmle und Wedde 2018). Zugleich aber werden Telearbeit und mobile Arbeit als Chance für Zugewinne an Arbeitszeitautonomie und eine Verbesserung der Vereinbarkeit von Beruf und Privatleben betrachtet (BMAS 2017).

\section{Digitalisierungsfolgen und digitaler Kapitalismus}

Die zuvor angeführten Befunde und Hypothesen zeigen, dass es Belege für einheitliche und eindeutige Digitalisierungsfolgen mit Blick auf Arbeit nicht gibt. Vielmehr sind widersprüchliche Entwicklungstendenzen zu beobachten. Dazu gehören zwar auch (Re-)Taylorisierung und Kontrolle, zugleich aber auch Requalifizierung und Zugewinne an Autonomie. Diese Heterogenität lässt sich mit Rückgriff auf zwei arbeitssoziologische Binsenweisheiten besser verstehen, die in der Debatte nicht immer berücksichtigt werden: Dass erstens Digitalisierung zwar ein wichtiger, aber keinesfalls der einzige Entwicklungstrend ist, der die Entwicklung von Arbeit aktuell prägt; und dass zweitens die konkrete Gestaltung, Organisation und Regulierung von Arbeit Gegenstand von betrieblichen, aber auch überbetrieblichen Arbeitspolitiken ist und damit das Ergebnis der Interaktionen von Akteuren wie Management und Arbeitgeberverbänden sowie Gewerkschaften und Betriebsräten und ihren Deutungen, Interessen, Strategien und Machtressourcen (siehe hierzu auch Lehndorff 2006).

Im aktuellen Diskurs finden sich viele Versatzstücke eines technologiegeprägten Verständnisses kapitalistischer Entwicklung, die als weitgehend autonom von anderen Veränderungen und von arbeitspolitischen Einflüssen betrachtet wird. Beispielhaft dafür sei das Leitbild der Industrie 4.0 genannt. Hier wird eine Abfolge von technologisch bestimmten industriellen Revolutionen gezeichnet: Dampfkraft in der ersten, Fließband und Elektrifizierung in der zweiten, IT und Elektronik in der dritten und schließlich Cyber-Physische Systeme in der vierten industriellen Revolution sind die Kerntechnologien, die demnach die langfristigen Entwicklungstendenzen der wirtschaftlichen Dynamik geprägt haben oder prägen werden. Grundlage dafür sind demnach jeweils neuartige Produktivitätsund damit Profitspielräume, die mit der Nutzung der Basistechnologien einhergehen. Nicht von ungefähr wurde mit der Digitalisierung die Hoffnung auf einen Produktivitätsanstieg von $30 \%$ verbunden (BMWi 2015).

Diese Zuweisung einer zentralen Rolle an die Technik als Triebkraft kapitalistischer Entwicklung erinnert an die Theorie der langen Wellen kapi- 
talistischer Entwicklung, die vom russischen Ökonomen Kondratjew in den 1920er Jahren entwickelt wurde (Kondratjew 1926); er ging davon aus, dass jeweils technologische Basisinnovationen lange Konjunkturzyklen begründen, die den klassischen kurzfristigen Konjunkturzyklus überlagern. Schumpeter hat schließlich das Konzept der technologiegetriebenen langen Wellen aufgenommen und sie mit einer zentralen Rolle des kapitalistischen Unternehmers verbunden, der als dynamischer Faktor Inventionen zu Innovationen macht und damit nicht nur die Produktmärkte in Bewegung bringt, sondern auch die Arbeits- und Kapitalmärkte (Schumpeter 1961).

Diese Sichtweise kapitalistischer Entwicklung ist von den Verfechtern digitalen Wandels wiederaufgenommen worden. Rifkin (2014) beispielsweise prognostiziert mit dem Internet der Dinge einen „extremen Produktivitätssprung“, der durch die damit verbundene smarte Infrastruktur erzeugt würde, nämlich die Vernetzung von Maschinen, Fabriken und Unternehmen, aber auch Häusern und Fahrzeugen. Auf diese Weise könnten über das Internet Kommunikation, Energie und Logistik als Triebkräfte der Produktivität verbunden werden. Mehr noch, durch digitale Verbreitung von Waren würden die Grenzkosten der Produktion gegen Null tendieren und damit die Profite als Quelle der Kapitalakkumulation reduzieren und die kapitalistische Warenproduktion auf immer kleineren $\mathrm{Ni}$ schen eindämmen; im Gegenzug würden neue Formen des gemeinsamen Wirtschaftens, die „Collaborative Commons“, entstehen, deren neue Formen der Zusammenarbeit in der "Sharing Economy“ durch das Internet als globale Plattform ermöglicht werde. Auf diese Weise werden Produzent*innen und Konsument*innen in eine Person vereint, die „Prosumers“, die Informationen, grüne Energie, Produkte (durch 3-D-Druck erzeugt) oder auch Häuser, Fahrzeuge und Dienstleistungen tauschen. Ähnlich argumentiert Mason (2015) mit einem technologisch getriebenen Postkapitalismus; wie bei Rifkin (2014) wird ein Widerspruch zwischen einer möglichen Gesellschaft freier und im Überfluss produzierter Güter auf der einen und dem aktuellen kapitalistischen Wirtschaftssystem auf der anderen Seite festgestellt und prognostiziert, dass die Tendenz sinkender Grenzkosten den Kapitalismus sowohl technologisch unterminiere als auch den Postkapitalismus als politisches Projekt in eine realistische Reichweite bringe.

Allerdings ist die Vision der Sharing Economy (dazu Sundararajan 2016), die auf neuen Beteiligungsformen durch gemeinsamen Austausch und gemeinsamer Produktion in Open-Source-Prozessen - und nicht auf abhängiger Beschäftigung im Lohnverhältnis - beruht, deutlich unterkomplex. Schon Schiller (1999), der als erster den Begriff des digitalen Kapita- 
lismus prägte, hat festgestellt, dass der Cyberspace nach seiner Entwicklung durch Staat, Militär und Bildungsinstitutionen in den USA durch große Konzernen dominiert wird. Gerade auch die Internetindustrie im engeren Sinne ist durch starke Unternehmenskonzentration bei den großen amerikanischen und mittlerweile auch chinesischen Internetkonzernen (Staab und Butollo 2018), laufende Restrukturierungen der Geschäftsportfolios und die Suche nach neuen Geschäftsfeldern geprägt (Dolata 2015). Dezentrale Marktstrukturen und demokratische Produktions- und Innovationsprozesse sind demnach allenfalls von marginaler Bedeutung. Es sind die großen Internetkonzerne, welche die technische Infrastruktur betreiben und entwickeln und damit entscheidenden Einfluss auf die Strukturierung und Regulierung - beispielsweise von Zugängen und Inhalten - haben (Dolata 2020). Hervorzuheben ist, dass dies nicht allein auf die ökonomische Sphäre beschränkt ist, sondern auch für private Nutzungen des Internets gilt. So sind die zu beobachtenden Digitalisierungsprozesse nicht als rein produktionsbezogen zu charakterisieren, sondern stellen vielmehr einen umfassenden gesellschaftlichen Digitalisierungsprozess dar. Damit ist zugleich ein „Prozess der kommerziellen Vermessung und Kommodifizierung sozialer Aktivitäten" (Dolata 2020, S. 184) verbunden, der in dieser Form und in diesem Ausmaß eine neue Qualität darstellt.

Die Visionen sind allerdings nicht nur wegen der Ausblendung von Markstrukturen unterkomplex, sondern auch wegen ihrer Vernachlässigung sozialer und politischer Institutionen und sozialer Akteure mit jeweils eigenen Deutungen, Interessen und Machtressourcen. Die damit einhergehende Komplexität der Analyse kapitalistischer Entwicklung lässt sich anhand der Fordismusanalyse aufzeigen, mit deren Hilfe Vertreter der Regulationstheorie die Wirtschaftswunder nach dem zweiten Weltkrieg in den entwickelten politischen Ökonomien zu erklären versucht haben ( $\mathrm{Li}$ pietz 1985). Dazu zählt zum einen die Entwicklung von Produktionssystemen (Boyer und Freyssenet 2003) der Massenproduktion auf der Grundlage technologischer Innovationen wie dem Fließband, arbeitsorganisatorischer Neuerungen wie dem Taylorismus, Neuausrichtungen der Markt und Produktstrategien aus Massenprodukte und sozialer Innovationen steigender Löhne und einer Anerkennung der Gewerkschaften. Hinzu kamen zum anderen institutionelle und politische Faktoren wie die Etablierung nationaler Systeme der industriellen Beziehungen und - zumeist überbetrieblicher Kollektivverträge, die Entwicklung bzw. den Ausbau sozial- und wohlfahrtstaatlicher Leistungen, die Einführung einer keynesianischen Wirtschaftspolitik und ein durch das Weltwährungssystem von Bretton Woods aufgespannter weltwirtschaftlicher Rahmen, der Freiräume für national geprägte Wirtschafts- und Wohlfahrtspolitiken schuf, indem 
er kurzfristige Finanzströme auf den Kapitalmärkten unterband. Erst auf Grundlage dieser Regulierungen und eines kohärenten Ensembles von Institutionen sozialer Praktiken konnte eine längerfristige Entsprechung von Massenproduktion und Massenkonsum als Kern des fordistischen Akkumulationsregimes geschaffen werden. Technologie, sei es in Form des $\mathrm{Zu}$ gangs zu Elektrizität oder des Fließbands oder Einzweckmaschinen, spielte eine ermöglichende Rolle, doch erklärt sie nicht die Entwicklungen von Institutionen und Praktiken; diese sind nur als Ergebnis sozialer Kompromisse und Konflikte zu verstehen.

Ähnliches gilt auch für die daran anschließende Debatte um den Postfordismus (Lipietz 1997) oder die flexible Spezialisierung (Piore und Sabel 1985). Hier stand die Flexibilisierung der Produktion im Zentrum, die auf einer stärkeren Nutzung von fachlichen Qualifikationen und Produzentenwissen in neuen Produktionskonzepten (Kern und Schumann 1984) beruhte. Auch dabei spielten neue technologische Entwicklungen wie numerische Steuerungen eine Rolle, weil sie die Flexibilität des Maschineneinsatzes zumindest potenziell erhöhten. Die Ausbreitung neuer Produktionskonzepte, wie beispielsweise der Gruppenarbeit, ist aber letztlich ohne die Analyse sozialer Konflikte und Kompromisse in den Betrieben nicht zu verstehen.

\section{Verschränkung der Digitalisierung mit anderen Entwicklungstrends}

Doch die Unterbelichtung sozialer Komplexität ist nicht das einzige Problem der Visionen eines digitalen (Post-)Kapitalismus. Ein zweites Problem ist die Ausblendung anderer Entwicklungstrends im aktuellen Kapitalismus mit mehr oder weniger starken Auswirkungen auf die Arbeit. Davon sind an dieser Stelle zwei Entwicklungen anzusprechen: die Globalisierung der Produktion und die Finanzialisierung der Corporate Governance (Haipeter 2016). Andere Entwicklungen wie die staatliche Arbeits-, Wirtschaftsund Sozialpolitik, demographische Verschiebungen oder Migration in Arbeitsmärkte könnten freilich ebenfalls angefügt werden.

Die Globalisierung der Produktion durch Direktinvestitionen multinationaler Unternehmen (MNU) in andere Länder ist zwar kein ganz neues Phänomen, doch hat sie sich in den letzten zweieinhalb Jahrzehnten in Umfang und Qualität deutlich verändert und ist zu einem zentralen Einflussfaktor des Wandels von Arbeit geworden. Das gilt zum einen wegen der weltweit starken Wachstumsdynamik ausländischer Direktinvestitionen und der deutlichen Zunahme der Zahl der MNU, zum anderen aber auch und vor allem wegen des Formenwandels ihrer Strukturen (Dicken 
2015). Produktives Kapital sucht inzwischen weltweit nach Marktzugängen und Arbeitskräften, letzteres mit Blick auf Qualifikationen und vor allem auf niedrige Löhne. Auf diese Weise bietet sich den Unternehmen die Möglichkeit eines „Regime-Shopping“ auf der Suche nach den besten Angebotsbedingungen von Arbeit und zugleich die Möglichkeit, durch Androhung von Produktionsverlagerungen Druck auf die Arbeitsregulierung und Arbeitsbedingungen ausüben zu können (Streeck 1998). Diese Möglichkeiten werden noch erweitert durch den Übergang von multinationalen zu globalen und transnationalen Konzernstrukturen (Hirsch-Kreinsen 2018c) und zu globalen Produktionsnetzwerken, in denen die Produktion mühelos zwischen unterschiedlichen Produktionsstandorten verschoben werden kann. Auf diese Weise entsteht eine Standortkonkurrenz um Beschäftigung, Produktion und Investitionen, die erhebliche Regulierungsprobleme für Arbeit aufwirft, die wirksam nur durch Koordinierung auf transnationaler Ebene angegangen werden können (Haipeter, Hertwig und Rosenbohm 2019). Zudem haben sich in vielen Branchen neue Formen der Hersteller-Zulieferbeziehungen in Form globaler Wertschöpfungsketten entwickelt (Gereffi, Humphrey und Sturgeon 2005), die aus Endherstellern, global aktiven Zulieferern und lokalen Zulieferern in mehrstufigen Strukturen bestehen und sich zumeist durch einen erweiterten Wertschöpfungsanteil der Zulieferer auszeichnen. Auch damit sind Probleme für die Regulierung und die Bedingungen von Arbeit verbunden, denn Zulieferunternehmen - und je mehr, desto niedriger ihre Wertschöpfungsstufe - weisen nicht selten schlechtere und prekäre Arbeitsbedingungen im Vergleich zur Arbeit an den Hauptsitzen der MNU in den entwickelten politischen Ökonomien auf (Doellgast und Gospel 2013). Auslagerung kann damit neben den Verlagerungen der Produktion zur Kostensenkung eingesetzt und als weitere Drohkulisse von den Unternehmen genutzt werden.

Die Finanzialisierung der Corporate Governance ist eng verbunden mit dem - zumindest bis zur Finanzmarktkrise - überaus dynamischen Wachstum der internationalen Finanzmärkte, dessen Volumen Welthandel oder Direktinvestitionen um ein Vielfaches übertraf (Dicken 2015). Hinzu kam die Schwächung alternativer Finanzierungsformen jenseits der Börse in vielen Ländern (wie in Deutschland die Abkehr vom bankbasierten Finanzierungssystem), die Entstehung neuer Finanzmarktakteure wie Investitions- und Pensionsfonds, Hedgefonds oder Private Equity Unternehmen mit zumeist kurzfristigen Renditeinteressen sowie auch die Bedeutungszunahme externer Kontrollformen und durch Investoren, sei es durch Einflussnahme auf Hautversammlungen oder durch Verkauf von Aktien an 
den Börsen, und die damit verbundenen Informations- und Berichterstattungsvorschriften (Windolf 2008).

Der Wandel der Kontrollformen für Investoren steht in unmittelbarer Beziehung zur Veränderung der Corporate Governance der Unternehmen und dem Unternehmensleitbild der Maximierung des Shareholder Value, das sich zunächst in den USA ausgebreitet hat (Lazonick und O'Sullivan 2000). Nicht mehr Wachstum durch Einbehaltung und Reinvestition der Unternehmensgewinne prägen dieses Leitbild, sondern Kostensenkung durch Abbau von Beschäftigung und die Neuverteilung der Gewinne zugunsten der Anteilseigner. Grundlage dafür war in den USA - und sich von dort ausbreitend - die Entwicklung eines finanzwirtschaftlichen Kontrollkonzepts in den Unternehmen, das sich durch drei Merkmale auszeichnet: erstens die Dezentralisierung operativer Managemententscheidungen in Geschäftsbereichen oder Divisionen, zweitens die Bewertung dieser Einheiten nach ihrer finanziellen Performance als entscheidendem Kriterium für ihren Kauf oder Verkauf und drittens der Bedeutungsgewinn der Finanzmanager*innen gegenüber den Manager*innen aus dem Produktionsbereich oder dem Vertrieb (Fligstein 1990). Diese Finanzialisierung der Unternehmenssteuerung als neues Leitbild der Unternehmensführung (Dörre und Haubner 2012) schuf eine Begründungsordnung, mit deren Hilfe Managementhandeln neu ausgerichtet und legitimiert werden konnte (Kädtler 2009; Froud, Johal, Leaver und Williams 2006), und zwar sowohl nach außen gegenüber den Finanzmärkten als auch nach innen gegenüber den Beschäftigten und ihren Interessenvertretungen eingesetzt, um finanzwirtschaftliche Vorgaben als Sachzwänge der Märkte darzustellen. Auf dieser Grundlage werden zum einen Leistungskennziffern vorgegeben oder Personal- und Sachbudgets definiert, die einen Rahmen setzen, der im Sinne einer indirekten Steuerung und stärkerer unternehmerischer Verantwortung der Beschäftigten (Glissmann und Peters 2002), aber auch im Sinne von mehr Kontrolle und Hierarchie genutzt werden kann. Ein zweiter Weg der Durchsetzung von Sachzwängen sind zum anderen Auslagerungen oder Desinvestitionen; nicht rentable Geschäftsfelder oder renditesenkende Kosten können durch Outsourcing oder Verkauf von Unternehmensteilen gesenkt werden. Zugleich können renditegetriebene Standort- und Auslagerungsentscheidungen als Druckmittel zur Verschlechterung der Arbeitsstandards, sei es durch Tarifabweichung, Tarifkonkurrenz, Tarifaustritt oder Prekarisierung der Beschäftigung, genutzt werden.

Bei alledem spielt die Digitalisierung eine wichtige Rolle als „Enabler“ aber nicht als determinierende Kraft. Die Digitalisierung hat die Globalisierung von Produktion und Finanzmärkten durch die Verbindung von Kommunikations- und Computertechnologien erleichtert. In diesem Sin- 
ne stellt sie also eine neuartige Infrastruktur bereit: Sie ist eine „time-space shrinking“ Technologie wie zuvor Dampfschiffe, die Eisenbahn, Düsenflugzeuge oder die Containertechnologie (Dicken 2015). Die Digitalisierung ergänzt diese physischen Transportmittel um den Transfer von Informationen. Digitale Technologien erlauben die Erfassung, Manipulation und Speicherung von Informationen weltweit und - zumindest nahezu in Echtzeit. Damit sind Informationen weltweit verfügbar. Investoren können ihr Kapital in Sekunden rund um den Globus transferieren, Unternehmen können globale Informationsräume schaffen, in denen Informationen zwischen ihren Standorten und mit anderen Unternehmen fließen, es können datenbezogene Standortvergleiche und Benchmarkings von Zulieferern durchgeführt werden, und Beschäftigte aus unterschiedlichen Ländern und Kontinenten (und Unternehmen) können an gemeinsamen Projekten arbeiten (Boes et al. 2015). Die Technologie schafft für diese vielfältigen Entwicklungen Möglichkeitsräume, aber sie entscheidet nicht darüber, ob und wie diese auch genutzt werden.

Zudem lassen sich auch Widersprüche zwischen den Entwicklungen feststellen. Die Finanzialisierung der Unternehmen kann nämlich auch ein Bremser für digitale Investitionen sein, sofern diese nicht die Ansprüche einer kurzfristig zu erzielenden Minimalverzinsung erfüllen (so auch Dörre 2017). Ähnlich kann sich die Globalisierung als Schranke für die Entwicklung der Digitalisierung in bestimmten Ländern oder an einzelnen Standorten erweisen, wenn digitale Investitionen nur an ausgewählten Standorten von MNU durchgeführt werden. Zwar ermöglicht die Digitalisierung eine dynamische Globalisierung von Produktion und Finanzmärkten, umgekehrt gilt dies aber nicht im gleichen Maße. Die Entwicklungen können sich wechselseitig verstärken, können aber auch konfligieren.

Zugleich entsteht ein zweites Problem, nämlich das der Zurechnung von arbeitspolitischen Folgen dieser Entwicklungen. Wenn in einem Betrieb Beschäftigte entlassen werden, liegt dies dann daran, dass ihre Arbeitsplätze automatisiert wurden, dass sie ins Ausland verlagert wurden oder dass sie nicht mehr rentabel - oder nicht mehr rentabel genug - sind? Oder wenn ein Großteil der Beschäftigten über einen Anstieg des Leistungsdrucks in digitalen Arbeitsprozessen klagt (DGB-Index 2016), ist dies dann ein zwangsläufiges Ergebnis eines bestimmten Umgangs mit digitaler Technik, also digitaler Arbeit oder der Digitalisierung der Arbeit, oder liegt dies womöglich daran, dass in den Bereichen mit stärkerem digitalen Technikeinsatz Strategien der Leistungsintensivierung durch Kennziffernsteuerung oder knappe Personalbudgets stärker zum Tragen kommen? Diese Fragen zeigen, wie schwierig es sein kann, die sich ergebenden Konsequenzen der Digitalisierung oder anderen Einflüssen zuzurechnen. Dies 
unterstreicht zugleich, dass die arbeitspolitischen Herausforderungen, die sich aktuell stellen, über die Digitalisierung im engeren Sinne hinausweisen. Wichtiger als die Zurechnung aber ist die Frage, ob und inwieweit es arbeitspolitische Antworten auf die tatsächlichen oder möglichen Probleme der Arbeitsbedingungen, der Arbeitsregulierung und Arbeitsgestaltung gibt, die sich derzeit abzeichnen und zu denen die Digitalisierung beiträgt.

\section{Digitalisierung und Arbeitspolitik}

Das Zurechnungsproblem führt dazu, dass sich die aktuellen arbeitspolitischen Herausforderungen nicht umstandslos der Digitalisierung zuweisen lassen. Häufig haben sie andere Ursachen, werden aber durch die Nutzung digitaler Technologien möglicherweise verstärkt oder auch abgeschwächt. Teilweise sind sie aber auch - wie neue Qualifikationsbedarfe im Umgang mit Software - durch die Digitalisierung hervorgerufen, werden aber durch andere Entwicklungen - finanzgetriebene Deckelung von Personal und Budgets - als Probleme verschärft. Dies ist deshalb ein Problem für die Arbeitspolitik, weil sich diesen Herausforderungen nicht dadurch begegnen lässt, nur den Technologieeinsatz und seine Folgen zu problematisieren oder zu beeinflussen.

Dazu sollen kurz drei Beispiele skizziert werden. Ein erstes Beispiel für eine Verstärkung wäre Mehrarbeit und lange Arbeitszeiten, die originär durch knappe Personalbemessungen aufgrund eng geschnittener Budgets und Kostenvorgaben verursacht werden. Dies begründet eine Personalpolitik der unteren Linie, die Unternehmen mit Verweis auf Standortkonkurrenz oder Renditevorgaben fahren und die vorsieht, Überschreitungen der Kapazität zunächst mit Mehrarbeit auszugleichen, dann möglicherweise flexible Erweiterungen durch Leiharbeit oder Auslagerungen zu nutzen und erst am Ende Neueinstellungen vorzunehmen. Ergänzend können lange Arbeitszeiten ihre Wurzeln in Dispositionen der Beschäftigten wie Leistungsorientierung, fachlicher Motivation oder Karrierezielen haben (Haipeter, Bromberg und Slomka 2016). Digitalisierung kann dann verstärkend wirken, wenn Beschäftigte neue Softwareprogramme einsetzen müssen, ohne dafür richtig geschult zu werden oder ohne, dass mögliche technischen Probleme neuer Softwareschnittstellen oder des Auslaufens der alten Programme gelöst werden, so dass technisch bedingte Mehrarbeit entsteht.

Ein zweites, eng damit zusammenhängendes Beispiel ist die zu beobachtende Zunahme der Arbeitsintensität und des Leistungsdrucks; auch hier wirken unterschiedliche Treiber zusammen. Ohne die Kenntnisnahme der 
Personalstrategien der unteren Linie wären diese Phänomene kaum erklärbar. Hinzu kommen Arbeitsorganisationsformen, die darauf abzielen, die „Poren des Arbeitstages“ zu schließen. Zu denken ist dabei an Ganzheitliche Produktionssysteme in der industriellen Fertigung, in deren Rahmen eine beschäftigtengetragene Optimierung von Standards erreicht werden soll (Clarke 2005) oder auf Projektstrukturen in hochqualifizierten Arbeitsbereichen mit knappen Budgets, unklaren Zuständigkeiten und mangelnder Anpassung an veränderte Bedingungen (Kalkowski und Mickler 2009). Digitalisierung schafft für solche Prozesse eine Infrastruktur, weil damit neue Standards verbreitet werden können oder Kommunikation im Projekt auch über räumliche und zeitliche Distanzen erfolgen kann; sie kann aber auch Prozesse verkomplizieren, wenn die Technik nicht problemlos funktioniert und auf diese Weise Mehrarbeit und Leistungsdruck erzeugen.

Drittens ist an die oben beschriebene Entwicklung des Outsourcings und der Verlagerung zu denken, für die digitale Technologien neue Möglichkeiten eröffnet. So ist das Offshoring von zentralen Funktionen in den Unternehmen wie Teilen der Sachbearbeitung beispielsweise in Controlling und Rechnungswesen oder auch im HR-Bereich technisch daran gebunden, dass die jeweiligen Prozesse digital bearbeitet und online und quasi in Echtzeit auch am räumlichen Sitz dieser Funktionen verfügbar sind. Digitale Datenverarbeitung ist wiederum durch die digitale Technologie zumindest potenziell gut kontrollierbar. Diese Liste ließe sich fortsetzen, auffällig sind indes die Interdependenzen der Digitalisierung mit anderen Treibern der Veränderungen von Arbeit. Insofern sind es auch nicht die eingesetzten Technologien an sich, welche die Veränderungen anstoßen, sondern der Einsatz (digitaler) Technologien ist eingebettet in historische Pfadverläufe, in denen sich ökonomische Interessen, politische Machtverhältnisse und kulturelle Werte spiegeln (Pfeiffer 2018, S. 321).

Was folgt daraus für die Arbeitspolitik unter den Vorzeichen der Digitalisierung? Die Beispiele zeigen, dass zumindest zwei arbeitspolitische Handlungsschwerpunkte für den Umgang mit Problemen der Arbeit und der Arbeitsbedingungen in den digitalen Arbeitswelten existieren. Der erste betrifft Probleme, die unmittelbar der Digitalisierung zugerechnet werden können. Dazu zählen beispielsweise die bereits erwähnten Anpassungsschwierigkeiten im Umgang mit neuen Programmen oder Schnittstellenprobleme zwischen Softwareprogrammen, aber auch Programme, die nicht auf die Tätigkeiten und Anforderungen der User*innen zugeschnitten sind und dadurch zusätzlichen Aufwand verursachen. $\mathrm{Zu}$ denken ist auch an unmittelbar durch die Nutzung digitaler Technologien entstehende Qualifikationsanforderungen und vor allem betriebliche Re- 
strukturierungen und Rationalisierungen, die sich aus dem digitalen Technologieeinsatz ergeben. Beispiele dafür wären die Ersetzung bestimmter Funktionen durch digitale Prozesse. So könnten Funktionen der Sachbearbeitung beispielsweise der Eingabe von Bestelldaten durch digitale Kundenaufträge ebenso ersetzt werden wie Teile der Arbeitsplanung durch die direkte Weiterleitung von Kundenaufträgen an Maschinen, und es stellt sich dann das Problem der Weiterbeschäftigung und Re-Qualifizierung der Beschäftigten für neue Aufgaben.

Hier beruhen die Chancen der Bewältigung der arbeitspolitischen Herausforderungen auf der Gestaltung von digitalisierter und digitaler Arbeit im engeren Sinne. Dort, wo digitale Technologien bei der Arbeit zur Anwendung kommen, sind individuelle, organisatorische und systemische Anpassungen vonnöten (Sträter 2019), um den Arbeitsprozess nachhaltig zu gestalten und nicht kurzfristigen Hypes entsprechend zu Lasten von Beschäftigten ,zurechtzubiegen' (Lehndorff 2006, S. 10). Solche Probleme, die der Einsatz digitaler Technologien für Beschäftigte nach sich zieht, sind nicht zuletzt in einer steigenden psychischen Belastung zu finden (Gerlmaier und Geiger 2018, S.2; Sträter 2019, S. 252). Um Arbeitnehmer*innen in die Lage zu versetzen, nicht nur mit den neuen an sie gestellten Anforderungen ,Schritt halten' zu können, sondern auch von den vielfach postulierten Chancen der Digitalisierung (Rothe, Wischniewski, Tegtmeier und Tisch 2019) profitieren zu können, ist es notwendig, bereits bei der Einführung von digitalen Technologien den Aspekt der (betrieblichen) Gestaltung von Arbeit zu berücksichtigen. Mit der Digitalisierung wird deshalb die Technologie und ihre arbeitsorganisatorische Gestaltung zu einem zentralen Thema der Arbeitspolitik. Dazu braucht es neue Leitbilder einer menschengerechten digitalen und digitalisierten Arbeit. Für solche Interventionen ist wiederum der Ausbau des Wissens über die Wirkungen von Digitalisierungsprozessen erforderlich (Parker und Grote 2019). Die Digitalisierung bedingt eine Veränderung der bisherigen „Muster von Ressourcen, Belastungen, Beanspruchungen und Teilhabemöglichkeiten" (Latniak und Gerlmaier 2019, S. 21) auf der betrieblichen Ebene, die jedoch bislang auf wenig Wissen bezüglich der Gestaltungskompetenzen - sowohl auf Seiten der Beschäftigten und ihrer Interessenvertretungen als auch der Führungskräfte und des Managements - trifft (Rothe et al. 2019, S. 249-250).

Der zweite Handlungsschwerpunkt kreist um die Probleme, die aus anderen Entwicklungen als der Digitalisierung erwachsen. Eine Konzentration nur auf die Digitalisierung würde verkennen, dass Probleme wie Gefährdungen von Beschäftigung, Standortschließungen und Verlagerungen, Personalstrategien der unteren Linie oder Arbeitsintensivierungen nach 
wie vor bestehen und zu guten Teilen andere Ursachen haben. Nur wenn auch diese Ursachen zum Thema der Arbeitspolitik gemacht werden, lassen sich neue Kompromisse und Lösungen erhoffen. Eine auf nachhaltige Entwicklung des individuellen Arbeitsvermögens setzende Arbeitspolitik darf sich deshalb nicht nur auf den Hype der Digitalisierung konzentrieren, freilich ohne die Probleme zu verkennen, die sich unmittelbar aus der Digitalisierung der Arbeit ergeben.

Zugleich aber ist dieser Hype dafür ein hilfreicher Anknüpfungspunkt. Auch jenseits des Zuschreibungsproblems arbeitspolitischer Herausforderungen kann es arbeitspolitisch sinnvoll sein, die vielfältigen arbeitspolitischen Herausforderungen und Handlungsbedarfe unter der Überschrift der Digitalisierung zu behandeln. Der Grund dafür liegt auf der Hand: Die Digitalisierung erhält aktuell hohe Aufmerksamkeit, und so können arbeitspolitische Probleme in den Fokus kommen und gegebenenfalls skandalisiert werden, auch wenn sie nur mittelbar auf die Digitalisierung zurückzuführen sind. Langfristige Probleme der Arbeitsbedingungen können so in die öffentliche Wahrnehmung gerückt werden, die sonst weniger Beachtung fänden. Deshalb auch sind die Gewerkschaften in der Debatte um Digitalisierung überaus aktiv und versuchen, sich in die politischen Prozesse einzubringen. Das erklärte Ziel dabei lautet, Prozesse zu gestalten und mitzuprägen, um mögliche negative Folgewirkungen der Digitalisierung mit Blick auf Beschäftigung oder Arbeitsbedingungen abzuwenden und Chancen der Digitalisierung für eine Verbesserung der Arbeitsbedingungen, beispielweise mit Blick auf die Automatisierung von physisch belastenden Tätigkeiten oder eine Erweiterung von Autonomiespielräumen, systematischer nutzen zu können.

Eine zentrale Voraussetzung einer solchen Politik der guten Arbeit unter den Bedingungen der Digitalisierung sind Akteure, die diese Politik entwickeln und umzusetzen versuchen. Dazu zählen in erster Linie Gewerkschaften und Betriebsräte, aber auch die Beschäftigten als Expert*innen ihrer Arbeit, Führungskräfte sowie Personal- und Unternehmensleitungen, die eine langfristige Entwicklung des Arbeitsvermögens ihrer Beschäftigten im Auge haben, und schließlich Beratungsunternehmen oder andere Akteure der Arbeitsgestaltung, die Interessenvertretungen und Unternehmen dabei beraten und unterstützen können. Gewerkschaften und Betriebsräte haben erste Schritte einer aktiven Arbeitspolitik der Digitalisierung unternommen, die auf mehreren Handlungsebenen zu beobachten sind. Auf der Ebene der Politik wirken die Gewerkschaften an den Programmen der Bundesregierung, insbesondere der Plattform 4.0 und den damit verbundenen Prozessen mit, aber auch an Initiativen auf Länderebene wie der „Initiative Wirtschaft und Arbeit 4.0 in NRW“; hier sind durch- 
aus Ansatzpunkte eines neuen, thematisch fokussierten Korporatismus zu erkennen. Auf der Tarifebene gibt es gemeinsame Erklärungen mit den Arbeitsgeberverbänden wie die von Metall NRW und der IG Metall aus dem Jahr 2015 mit dem Titel „Digitalisierung, Industrie 4.0 und Arbeit 4.0“, in der sie sich zu einer „arbeitspolitische(n) Begleitung der Digitalisierung durch die Tarifvertragsparteien" bekennen und dazu mehrere Themenfelder wie Qualifizierung, Arbeitszeit, Entgelt oder Arbeits- und Gesundheitsschutz benennen. Zugleich starteten die Gewerkschaften konkrete betriebliche Projekte einer aktiven Gestaltung der Digitalisierung in den Betrieben, so die IG Metall mit ihrem Vorstandsprojekt „Arbeit und Innovation 4.0“ und ihrem Projekt „Arbeit 2020" der IG Metall NRW, in denen jeweils in unterschiedlicher Form Beratungs- und Qualifizierungsangebote für Betriebsräte gemacht werden, die jeweils auch darauf abzielen, das Expertenwissen der Beschäftigten für die Interessenvertretungen als Ressource nutzbar zu machen (zum Projekt Arbeit 2020 siehe Haipeter, Korflür und Schilling 2018). Mit diesen Erfahrungen wurde Digitalisierung dann von der IG Metall in einen breiteren strategischen Rahmen der Transformation eingebunden, in den daneben auch sozial-ökologische Herausforderungen einfließen. Ver.di bearbeitet weniger konkrete Projekte, bietet aber zum Thema bundesweit Seminare an und veranstaltet einmal im Jahr einen Digitalisierungskongress. Die IG BCE hat Handlungshilfen und Materialien für die Betriebsräte sowie Faktenblätter und Brancheninfos veröffentlicht. Gesonderte Projekte existieren für die Plattformökonomie. Die IG Metall hat in Zusammenarbeit mit Gewerkschaften anderer europäischer Länder die Plattform „faircrowdwork“ eingerichtet, auf der Crowdworker*innen Ratings zu Plattformen angeben können und einen Link zu einer gewerkschaftlichen Telefonhotline für Crowdworker*innen finden. Ver.di setzt in diesem Zusammenhang auf individuelle Beratung und nutzt dafür die Beratungsdienstleistungen, die sie traditionell für Selbstständige (hier vor allem Journalisten) anbietet.

Insgesamt also gibt es nicht wenige gewerkschaftliche Initiativen zur aktiven Einflussnahme auf die Gestaltung der Digitalisierung. Doch ist der Gestaltungsanspruch das eine, das strategische Handlungsvermögen der Gewerkschaften das andere. Dazu gehören organisatorische, personelle und finanzielle Ressourcen ebenso wie die Fähigkeit, Deutungen und Ziele zu entwickeln, Interessen zu artikulieren oder auch die Beteiligung der Mitglieder und Beschäftigten als Ressourcen zu nutzen (Lévesque und Murray 2013). Vor allem schließt dies auch die Frage von Präsenz und Organisationsmacht ein. Wo es keine Betriebsräte gibt und die Gewerkschaften nicht in den Betrieben verankert sind, finden arbeitspolitische Prozesse ohne die Unterstützung kollektiver Organisationsmacht statt. Digitalisie- 
rung ist in diesem Fall ein Ergebnis betrieblicher Mikropolitiken zwischen unterschiedlichen Managementebenen und -fraktionen, möglicherweise auch besonders strukturmächtiger Beschäftigtengruppen, nicht aber ein Gegenstand der Arbeitsbeziehungen, weil dafür auf Beschäftigtenseite die kollektiven Akteure fehlen.

Die Entwicklung der Verbreitungsgrade von Tarifbindung und Betriebsräten als Näherungswerte für die Existenz kollektiver Interessenvertretungen im Betrieb spricht eine eindeutige Sprache. Laut den Daten des IAB-Betriebspanels (Ellguth und Kohaut 2020) waren im Jahr 2019 nur noch $27 \%$ aller Betriebe und $52 \%$ aller Beschäftigten - darunter $44 \%$ im Flächentarifvertrag - tarifgebunden. In einem Betrieb mit Betriebsrat arbeiten $41 \%$ (West) bzw. 36\% (Ost) der Beschäftigten; insgesamt weisen $9 \%$ der Betriebe einen Betriebsrat auf (Ellguth und Kohaut 2020). Der Anteil der Beschäftigten, die in einem Betrieb arbeiten, der sowohl einem Branchentarif unterliegt als auch einen Betriebsrat besitzt, liegt bei $24 \%$ (West) bzw. $14 \%$ (Ost). Damit arbeitet nur eine Minderheit der Beschäftigten in der sogenannten Kernzone der Arbeitsbeziehungen (Ellguth und Kohaut 2020). Hinzu kommt eine ungleiche Verteilung zwischen Branchen und Betriebsgrößenklassen. Danach finden sich die Institutionen der Arbeitsbeziehungen durchgängig in Großbetrieben im Verarbeitenden Gewerbe oder in der Energiewirtschaft, sind aber in kleineren Betrieben vor allem in den privaten Dienstleistungen, aber auch im Verarbeitenden Gewerbe, kaum mehr vorhanden. Daraus wiederum erwachsen erhebliche Probleme für die Arbeitspolitik der Interessenvertretungen. Gewerkschaftliche Projekte zur Gestaltung der Digitalisierung greifen faktisch nur in der Kernzone der Arbeitsbeziehungen, also in den Betrieben, in denen sie, und mit ihnen die Betriebsräte, präsent sind und noch über eine Organisationsmacht verfügen, die ihnen druckvolles Handeln auf den verschiedenen Handlungsebenen von der Politik bis zum Betrieb ermöglicht; ansonsten fehlen die betrieblichen Adressaten für eine kollektive Regulierung.

In jedem Fall sind dabei arbeitspolitische Akzente gefragt, mit denen auf der einen Seite neue Gestaltungsansprüche an die Technologie formuliert und neue Leitbilder einer guten digitalen und digitalisierten Arbeit entwickelt werden, die auf der anderen Seite aber auch die engeren Grenzen der Digitalisierung überschreiten, das Zusammenspiel der aktuellen arbeitspolitischen Herausforderungen in den Blick nehmen und zugleich die Stärkung der Organisationsmacht von Gewerkschaften und Betriebsräten anstreben. Arbeitspolitik in Zeiten der Digitalisierung muss nicht nur daraus ausgerichtet werden, neue Themen zu entwickeln und mit alten aber nach wie vor relevanten - Themen zu verbinden, sondern auch darauf, dabei die Handlungsfähigkeit der Akteure der Arbeitspolitik - näm- 
lich der Interessenvertretungen und der Beschäftigten selber - zu stärken. Auf diese Weise könnte der Hype um die Digitalisierung möglicherweise von den Interessenvertretungen strategisch dazu genutzt werden, neue Fundamente für eine Arbeitspolitik einzuziehen, deren Grundlagen so zukunftsfest sind, dass sie über die aktuelle Digitalisierungsdebatte hinauswirken. Deshalb ist eine zukunftsträchtige Arbeitspolitik in der Digitalisierung zugleich eine Arbeitspolitik über die Digitalisierung hinaus.

\section{Arbeitspolitische Entwicklungslinien und die Beiträge dieses Bandes}

Die Forschungsabteilung Arbeitszeit und Arbeitsorganisation (AZAO) des Instituts Arbeit und Qualifikation, deren aktuelle Mitglieder an diesem Buch mitgewirkt haben, arbeitet seit langer Zeit an der Schärfung des Konzepts der Arbeitspolitik. Dies geschieht zum einen in der täglichen Forschungs- und Projektarbeit, zum anderen aber auch in Form gemeinsamer Publikationen. Die konzeptionellen Grundlinien dafür wurden in unserem ersten Buch über „das Politische in der Arbeitspolitik“ gelegt (Lehndorff 2006), das sich insbesondere mit den Herausforderungen auseinandersetzte, die von der Vermarktlichung von Organisationen für die Arbeitspolitik ausging und noch immer ausgeht. Zumindest drei der dort formulierten Überlegungen haben sich als tragfähiges Gerüst des Begriffs der Arbeitspolitik erwiesen.

Dazu gehört erstens, Arbeitspolitik als „Netz von Politik“ zu verstehen, das sowohl in als auch jenseits von Betrieben relevant wird. Konkrete Arbeitsgestaltung und -regulierung findet in den Betrieben statt, aber sie hängt $\mathrm{ab}$ von überbetrieblichen Institutionen und Politiken, die von der Finanzmarktregulierung über die Arbeitsmärkte bis hin zur Politik der Reproduktion reichen. Betriebliche Arbeitspolitik ist damit zwar nicht von außen determiniert, steht aber mit ihren Themen, Ressourcen und Akteuren in Artikulationsbeziehungen zu politischen Prozessen höherer Handlungsebenen. Dabei kann zweitens das Leitbild der Nachhaltigkeit einen inhaltlichen Kompass für die Arbeitspolitik bieten. Nachhaltigkeit wird von uns als Orientierung an der langfristigen Nutzung, Erhaltung und Entwicklung des individuellen Arbeitsvermögens und des gesellschaftlichen Beschäftigungspotenzials verstanden. Nicht nur soll Arbeit gesundheits- und lernförderlich gestaltet sein, sondern es soll jeder und jedem ermöglicht werden, an dieser Arbeit auch zu partizipieren. Drittens schließlich zielt Arbeitspolitik darauf ab, den Beschäftigten Haltegriffe und Leitplanken zu geben, die ihre Handlungsressourcen verbessern, um nachhaltige Arbeitsbedingungen auch aktiv einzufordern. Arbeitspolitik zielt des- 
halb nicht auf umfassende Schutzprojekte, sondern auf eine „Hilfe zur Selbsthilfe", die Beschäftigte in ihren Autonomieansprüchen unterstützt.

Diese Sichtweise auf Arbeitspolitik setzte von Beginn an auch eine bestimmte Orientierung kollektiven Handelns von Interessenvertretungen voraus. Im Zentrum dieser Orientierung steht nicht die - in früheren Jahren für Betriebsräte wie Gewerkschaften durchaus übliche - Stellvertreterorientierung, sondern die Aufnahme der konkreten Interessen der Beschäftigten in die Vertretungspolitik. Dieser Aspekt gewann in den letzten Jahren an Bedeutung. Mehr noch, die Einbindung und Beteiligung von Beschäftigten und Mitgliedern in und an der Politik der Interessenvertretungen zeichnete sich zunehmend als Grundlage ihrer Revitalisierung ab und damit als Voraussetzung dafür, als zentrale Akteure der Arbeitspolitik neue Kraft zu schöpfen. Gerade unter den Bedingungen des Finanzmarktkapitalismus, den wir in unserem letzten Buch (Haipeter, Latniak und Lehndorff 2016) behandelt haben, erscheint die enge Verzahnung individuellen und kollektiven Interessenhandelns als wichtige Voraussetzung dafür, Arbeitspolitik an den „Brechungen“ zwischen Finanzmarktimpulsen und Unternehmensstrategien oder zwischen Unternehmenssteuerung und Arbeitsorganisation wirksam zu beeinflussen (Haipeter 2016).

Digitalisierung stellt die nachhaltige Arbeitspolitik zwar vor die oben beschriebenen neuen Herausforderungen, eröffnet aber auch neue Chancen für die arbeitspolitischen Akteure. Dies liegt nicht zuletzt an dem konkreten Einsatz digitaler Technik in der Arbeit, der die Digitalisierung auszeichnet. Vermarktlichung oder Finanzmarktkapitalismus sind Prozesse, die zwar Auswirkungen auf Arbeit und Arbeitsprozesse haben - deshalb wurden sie ja auch Gegenstände der Arbeitspolitik -, die aber selber wenig konkrete arbeitspolitische Anknüpfungspunkte bieten, weil sie - vom Blickwinkel der Arbeitsorganisation aus - abstrakt sind und ihre Auswirkungen auf die Arbeit nicht genau bestimmbar sind oder erst mühevoll analysiert werden müssen. Dies ist bei der Digitalisierung anders. Dass Digitalisierung Arbeit, ihre Organisation, ihre Qualifikationsanforderungen und ihre Bedingungen verändern wird, leuchtet unmittelbar ein, auch ohne dass erst eine wissenschaftliche Analyse dieser Prozesse zu Rate gezogen werden müsste. Sie ist konkreter erfahrbar und weniger vermittelt und damit besser politisierbar. Und hinzu kommt noch ein weiteres: Die Digitalisierung ist als Thema von Politik, Arbeitgeberverbänden und Unternehmen gesetzt worden. Während über Vermarktlichung und die Rolle von Finanzmarktorientierungen kaum jemand öffentlich gesprochen hat, ist die Digitalisierung als öffentliches Thema entwickelt worden, und zwar auch und insbesondere mit Blick auf ihre Auswirkungen auf Arbeit und, im Falle der Industrie 4.0, verbunden mit dem Versprechen, dass damit Ar- 
beit nicht nur flexibilisiert, sondern auch aufgewertet wird. Die Digitalisierung bietet damit für eine nachhaltige Arbeitspolitik eine neue Möglichkeit, diesen Diskurs aufzugreifen, zu beeinflussen und dabei Leitbilder einer nachhaltigen Arbeitspolitik in digitaler oder digitalisierter Arbeit zu entwickeln und einzufordern. Die Entwicklung und Vermarktung eines solchen Leitbildes im Diskurs dürfte von zentraler Bedeutung sein, um Frage der Arbeitsgestaltung und Organisation in der öffentlichen Diskussion zu verankern und damit die öffentliche Meinung zu prägen.

Mindestens genauso wichtig wie die Entwicklung überzeugender Leitbilder guter Arbeit ist jedoch die konkrete Arbeitspolitik in den Betrieben. Es sind diese betrieblichen Entwicklungen der Digitalisierung und die damit verbundenen Fragen einer nachhaltigen Arbeitspolitik, die im Zentrum der Beiträge dieses Bandes stehen. Das Buch versammelt Analysen und Forschungsergebnisse unserer Forschungsabteilung, die sich den arbeitspolitischen Herausforderungen der Digitalisierung aus unterschiedlichen disziplinären Perspektiven nähern: Arbeitssoziologische Betrachtungen finden sich darunter ebenso wie eher arbeitswissenschaftliche und arbeitspsychologisch ausgerichtete Beiträge. Die Fragestellungen der hier versammelten Beiträge reichen dabei von derjenigen nach den Gegenständen politischer Auseinandersetzungen und Kompromissbildungen am Arbeitsplatz über jene nach dem Stellenwert von Betrieben oder Unternehmen für die Arbeitspolitik bis hin zur Untersuchung von Handlungsmöglichkeiten auf überbetrieblicher Ebene. Trotz dieser Vielfalt der Betrachtungsweisen eint die Beiträge zum einen, dass sie die beobachteten Phänomene immer auch in Bezug auf andere Herausforderungen und Rahmenbedingungen von Arbeit analysieren, da sich die aktuellen arbeitspolitischen Herausforderungen nicht immer gänzlich und umstandslos der Digitalisierung zurechnen lassen. Zum anderen richten die Beiträge ihren Blick explizit auf die Arbeitspolitiken und ihre Perspektiven, die für das Verständnis der Gestaltung, Organisation und Regulierung von Arbeit essenziell sind.

Die Beiträge des Bandes richten sich an drei thematischen Schwerpunkten aus. Der erste Schwerpunkt betrifft die Auswirkungen der Digitalisierung auf die Organisation und die Frage, ob und wie sich dadurch altbekannte räumliche und zeitliche Grenzen der Organisation von Arbeit verschieben oder auch auflösen. In diesem Kontext gehen Erich Latniak und Sophie Rosenbohm der Frage nach, welchen Wandlungsprozessen die betriebliche Organisation der Produktion und der Arbeit unterliegt. Sie zeigen, dass Digitalisierung nicht alleiniger Treiber ist, sondern zusammen mit anderen Trends zu einer Veränderung von Regulierungskompetenzen und arbeitspolitischen Entscheidungs- und Gestaltungsvoraussetzungen 
beiträgt. Ihre Analyse verdeutlicht Konfliktlinien, an denen Kontroversen und Klärungsbedarf entstehen können und die arbeitspolitisch zu bearbeiten sind: die ,Binnenregulierung bezieht sich auf heterogene Betriebszugehörigkeiten und Belegschaften und stellt die Frage nach einheitlichen Arbeits- und Beschäftigungsstandards in diesen Kontexten. Ein analoges Problem - aber unter anderen rechtlich-organisatorischen Voraussetzungen - wird von ihnen unter ,Grenzregulierung' gefasst. Auch hier geht es um eine einheitliche und kollektive Regelung von Arbeits- und Beschäftigungsstandards, allerdings entlang unternehmensübergreifender Zusammenhänge, Konzernstrukturen und Wertschöpfungsketten. ,Prozessregulierung' fokussiert schließlich den Umgang mit neuen technisch-organisatorischen Systemen und die Frage, wie betroffene Beschäftigte hierbei beteiligt werden und ihre Interessen einbringen können. Dabei entstehen schrittweise neue Arbeitsstandards, wobei die Autor*innen erheblichen Klärungs- und Regelungsbedarf sehen, um die Digitalisierungspotenziale langfristig zu heben.

Eine für Organisationsgrenzen relevante Entwicklung stellt auch die verstärkte Nutzung von ortsflexiblem Arbeiten außerhalb des Betriebs dar. Jennifer Kaczynska und Angelika Kümmerling untersuchen daher in ihrem Beitrag, unter welchen Bedingungen Homeoffice und mobiles Arbeiten zum Einsatz kommt. Der Beitrag greift damit gerade in Zeiten der CoronaKrise des Jahres 2020 ein hoch aktuelles Thema auf. Während in der Forschung der Blick bislang vor allem stark auf Großbetriebe gerichtet wurde, rücken die beiden Autorinnen die Aufmerksamkeit explizit auf kleine und mittlere Unternehmen (KMU) und analysieren, welche Bedingungen gegeben sein müssen, damit Homeoffice und mobile Arbeit gelingen können. Mit Rückgriff auf den Ansatz der Zeitgeographie blicken sie auf die Hindernisse, auf die die Implementierung mobiler Arbeit und Homeoffice in KMU trifft. Vor dem Hintergrund aktueller Diskussionen um eine gesetzliche Regulierung konstatieren die Autorinnen, dass es einer genauen Passung zwischen Tätigkeitszuschnitten, den Anforderungen der Betriebe und nicht zuletzt der Bedürfnisse der Beschäftigten bedarf, damit ortsflexibles Arbeiten im Sinne einer nachhaltigen Arbeitspolitik funktioniert.

Der zweite Schwerpunkt rückt die Verbindung von Digitalisierung und Arbeitsbeziehungen und die Frage, wie Interessenvertretungen auf diesen Wandel reagieren und welche neuen Organisierungs- und Beteiligungsstrategien sie dabei einschlagen, aber auch, wie sie selber die neuen Möglichkeiten digitaler Kommunikation und Vernetzung nutzen, in den Fokus. Thomas Haipeter behandelt in seinem Beitrag das gewerkschaftliche Projekt „Arbeit 2020“, ein Vorreiterprojekt einer aktiven und beteiligungsorientierten Arbeitspolitik in der Digitalisierung. Er untersucht, welche Rolle 
die Beteiligung der Beschäftigten als Expert*innen ihrer Arbeit spielt, welche arbeitspolitischen Themen von den Akteuren entwickelt werden und unter welchen Bedingungen es gelingen kann, zukunftsweisende Vereinbarungen mit den Unternehmensleitungen zu treffen, in denen Beteiligungsrechte an der Gestaltung und Regulierung der Digitalisierung verankert werden. Für die Perspektiven der Arbeitspolitik in der Digitalisierung ergeben sich daraus drei wichtige Erkenntnisse. Erstens verschafft die Beteiligung der Beschäftigten als Expert*innen den Interessenvertretungen einen Wissens- und Legitimationsvorsprung über aktuelle Entwicklungen der Digitalisierung. Zudem zeigt sich zweitens, dass damit ein neues Selbstverständnis von Betriebsräten als proaktiven Akteuren verbunden ist, die eine strategische Ausrichtung nicht nur mit Blick auf die Behandlung der Digitalisierung, sondern auch auf die Entwicklung ihrer Kompetenzen und Ressourcen entwickeln können. Und drittens schließlich wird deutlich, dass die Erarbeitung arbeitspolitischer Konzepte gerade in kleineren Betrieben wesentlich von einem intensiven Zusammenspiel zwischen Gewerkschaften und Betriebsräten profitiert, in dessen Rahmen Betriebsräte für neue arbeitspolitische Themen aktiviert werden.

Anhand von Plattformarbeit - und damit einem Bereich von im Zuge der Digitalisierung nicht mehr betrieblich organisierter Arbeit - diskutieren Fabian Hoose und Thomas Haipeter in ihrem Beitrag mögliche Ansatzpunkte für eine aktive Interessenvertretungspolitik und Organisierung. Ausgehend von der Beschreibung grundlegender Herausforderungen von Plattformarbeit - hervorzuheben ist hier zum Beispiel die Abhängigkeit von einseitig bestimmten Plattformregeln, der häufig Unsicherheit begründende Beschäftigungsstatus der Plattformarbeitenden oder die bestehende Atomisierung, die ein gegenseitiges Wahrnehmen und Herausbildung kollektiver Interessen erschwert - zeigen sie anhand von vier Beispielen, wie sich dennoch Initiativen für eine aktive Interessenvertretungspolitik und Organisierung herausbilden. Ihre Analyse unterstreicht, dass der Bereich der Plattformarbeit zwar insgesamt vorrausetzungsvoll für die Artikulation kollektiver Interessen ist, aber die konkurrenzfördernde Plattformlogik das Entstehen gemeinsamer Interessen und Anliegen nicht prinzipiell ausschließt.

Im dritten Beitrag dieses Abschnitts untersucht Christine Üyük die Chancen und Herausforderungen, die sich aus der Nutzung digitaler Kommunikationsmedien für Interessenvertretungen in Multinationalen Unternehmen ergeben. In diesem Rahmen analysiert sie transnationale Protestaktionen und geht der Frage nach, welche Rolle digitale Informations- und Kommunikationsmedien für die Protestformierung und Mobilisierung spielen. Gibt es für Interessenvertretungen Anlass, von transnationalen so- 
zialen Bewegungen zu lernen, denen es mit Hilfe digitaler Medien bereits gelungen ist, solche Protestaktionen durchzuführen? Anhand von drei der seltenen empirischen Beispiele transnationaler Protestaktionen wird gezeigt, dass bei diesen Protesten der Einsatz digitaler Medien nicht so eindeutig zu beurteilen ist. Die Befunde legen die Schlussfolgerung nahe, dass der wichtigste Einflussfaktor für Art und Umfang der Nutzung digitaler Medien das Vorhandensein transnationaler Interessenvertretungsstrukturen ist. Wo es solche Strukturen gibt, können die Akteure auch bewährte analoge Formen der Information und Kommunikation nutzen, um Konflikte zu rahmen und die Belegschaften zu mobilisieren. Wo sie allerdings nicht existieren, sind digitale Medien als Kommunikationsbasis unverzichtbar. Aber auch dann ist der Erfolg nicht garantiert, sondern hängt von Zusammensetzung und Interessenlagen der Belegschaften ebenso ab wie von der Mächtigkeit der Unternehmensseite.

Der dritte Schwerpunkt des Bandes widmet sich schließlich den Perspektiven der Arbeitsgestaltung unter den Vorzeichen der Digitalisierung und der Frage, welche Gestaltungspotenziale erkennbar sind und mit welchen Strategien sie umgesetzt werden können. Anja Gerlmaier befasst sich in ihrem Beitrag mit der Frage, ob die aktuellen Digitalisierungsbestrebungen in der Produktion arbeitspolitisch genutzt werden können, um die psychische und physische Gesundheit der dort Beschäftigten zu stärken. Sie zeigt anhand von drei Fallbeispielen aus Produktionsbereichen mit überwiegender Anlerntätigkeit, Facharbeit und produktionsnaher Wissensarbeit auf, dass die Einführung neuer Digitaltechnik auf dem shop floor häufig zu vermehrten Stress führt, obwohl Gestaltungspotenziale zur präventiven Arbeitsgestaltung vorliegen. Durch den Aufbau von kollektiver Gestaltungskompetenz bei operativen Führungskräften und ihren Teams kann in den dargestellten Gestaltungsbeispielen im Bereich der qualifizierten Facharbeit und produktionsnahen Wissensarbeit zwar die Gesundheitssituation erheblich verbessert werden. Dieser Befähigungsansatz stößt jedoch bei gering qualifizierten Tätigkeiten mit hohem Rationalisierungspotenzial an seine Grenzen, weil auf betrieblicher Ebene oft kein großes Interesse besteht, die Arbeitsfähigkeit dieser Beschäftigtengruppe nachhaltig zu sichern. Die Befunde machen deutlich, dass Digitalisierung insbesondere bei gering qualifizierten Tätigkeiten keineswegs automatisch zu sicherer, gesunder und lernförderlicher Arbeit führt und hier ein starker betrieblich verankerter institutioneller Arbeits-und Gesundheitsschutz vonnöten ist.

Alexander Bendel wirft in seinem Beitrag schließlich ebenfalls einen Blick auf die Digitalisierung in Industrieunternehmen und bietet Einblicke in einen konkreten betrieblichen Digitalisierungsprozess. In diesem 
Kontext analysiert er, wie eine partizipative, integrierte und ganzheitliche Gestaltung von IT-gestützten Arbeitssystemen aussehen kann. Sein Beitrag unterstreicht, dass Digitalisierungsprozesse nicht losgelöst von den jeweiligen Betriebskontexten und dem arbeitspolitischen Handeln der betrieblichen Akteure zu verstehen sind. Digitalisierungsmaßnahmen treffen auf marktgesteuerte Zusammenhänge in den Betrieben, die aufgrund ihrer allzu einseitigen Fokussierung auf betriebswirtschaftliche Kennzahlen menschengerechte Arbeitsgestaltung verhindern können. Umso wichtiger ist die Einrichtung von nicht-wertschöpfenden Reflexionsräumen für die Beschäftigten, in denen die Bedingungen gelingender, also arbeitnehmerfreundlicher Digitalisierung ausgehandelt werden können.

\section{Literatur}

Abel, J. (2018). Kompetenzentwicklungsbedarf für die digitale Arbeitswelt. FGW-Studie Digitalisierung von Arbeit. Düsseldorf: FGW.

Alt, R., \& Puschmann, T. (2016). Digitalisierung der Finanzindustrie. Grundlagen der Fintech Evolution. Berlin: Springer.

Arntz, M., Gregory, T., \& Zierahn, U. (2016). The Risk of Automation for Jobs in OECD Countries: A Comparative Analysis. OECD Social, Employment and Migration Working Papers No. 189. Paris: OECD.

Boes, A., Kämpf, T., Langes, B., \& Lühr, T. (2015). Landnahme im Informationsraum. Neukonstituierung gesellschaftlicher Arbeit in der „digitalen Gesellschaft“. WSI-Miteilungen, 88(2), 77-85.

Boes, A., Kämpf, T., Langes, B., \& Lühr, T. (2018). "Lean" und "agil" im Büro. Neue Organisationskonzepte in der digitalen Transformation und ibre Folgen für die Angestellten. Forschung aus der Hans-Böckler-Stiftung, Band 193. Bielefeld: transcript.

Bonin, H., Gregory, T., \& Zierahn, U. (2015). Übertragung der Studie von Frey/Osborne (2013) auf Deutschland. Kurzexpertise 57. Mannheim: Zentrum für Europäische Wirtschaftsforschung.

Bonin, H., \& Rinne, U. (2017). Omnibusbefragung zur Verbesserung der Datenlage neuer Beschäftigungsformen. Kurzexpertise im Auftrag des Bundesministeriums für Arbeit und Soziales. IZA Research Report No. 80. Bonn: IZA.

Boyer, R., \& Freyssenet, M. (2003). Produktionsmodelle. Eine Typologie am Beispiel der Automobilindustrie. Berlin: edition sigma.

Brynjolfsson, E., \& McAfee, A. (2014). The Second Machine Age. Work, Progress and Prosperity in a Time of Brilliant Technologies. New York: Norton. 
Bundesministerium für Arbeit und Soziales, Abteilung Grundsatzfragen des Sozialstaats, der Arbeitswelt und der sozialen Marktwirtschaft (BMAS) (Hrsg.) (2017). Weißbuch Arbeiten 4.0. Berlin: BMAS. Verfügbar unter: https://www.bmas.de/S haredDocs/Downloads/DE/PDF-Publikationen/a883-weissbuch.pdf;jsessionid=B 08C0D3C7E9CE6A490A5D908B4BF4AF9.delivery2-master?_blob=publication File\&v=1 [19.11.20].

Bundesministerium für Wirtschaft und Energie (BMWi) (2015). Industrie 4.0 und digitale Wirtschaft. Impulse für Beschäftigung, Arbeit und Innovation. Berlin: BMWi. Verfügbar unter: https:/www.bmwi.de/Redaktion/DE/Publikationen/In dustrie/industrie-4-0-und-digitale-wirtschaft.pdf?__blob=publicationFile \&v=5 [19.11.20].

Butollo, F., Ehrlich, M., \& Engel, T. (2017). Amazonisierung der Industriearbeit? Arbeit, 26(1), 33-59.

Clarke, C. (2005). Automotive Production Systems and Standardisation. From Ford to the Case of Mercedes-Benz. Heidelberg: Physica-Verlag.

Däubler, W. (2015). Internet und Arbeitsrecht. Web 2.0, Social Media und Crowdwork. Frankfurt: Bund-Verlag.

Degryse, C. (2016). Digitalisation of the economy and its impact on labour markets. Working Paper 2016.02. Brussels: ETUI. Verfügbar unter: https://www.etui .org/publications/working-papers/digitalisation-of-the-economy-and-its-impact-o n-labour-markets [04.11.2020].

Dengler, K., \& Matthes, B. (2019). Digitalisierung in Deutschland: Substituierbarkeitspotenziale von Berufen und die möglichen Folgen für die Beschäftigung. In R. Dobischat, B. Käpplinger, G. Molzberger \& D. Münk (Hrsg.), Bildung 2.1 für Arbeit 4.0? (S. 49-62). Wiesbaden: Springer.

Deutsche Akademie der Technikwissenschaften (acatech) (2013). Deutschlands Zukunft als Produktionsstandort sichern. Umsetzungsempfehlungen für das $\mathrm{Zu}$ kunftsprojekt Industrie 4.0. Abschlussbericht des Arbeitskreises Industrie 4.0. Frankfurt am Main: acatech. Verfügbar unter: https://www.acatech.de/publikati on/umsetzungsempfehlungen-fuer-das-zukunftsprojekt-industrie-4-0-abschlussbe richt-des-arbeitskreises-industrie-4-0/download-pdf?lang=de [19.11.20].

DGB-Index Gute Arbeit (2016). Der Report 2016. Wie die Beschäftigten die Arbeitsbedingungen in Deutschland beurteilen. Berlin: DGB.

Dicken, P. (2015). The Global Shift. Mapping the Changing Contours of the World Economy (7. Auflage). Los Angeles: Sage.

Doellgast, V., \& Gospel, H. (2013). Outsourcing and Human Resource Management. Cornell: Cornell University ILR School. Verfügbar unter: http://digitalco mmons.ilr.cornell.edu/articles/1217 [19.11.2020].

Dolata, U. (2015). Volatile Monopole. Konzentration, Konkurrenz und Innovationsstrategien der Internetkonzerne. Berliner Journal für Soziologie, 24(4), 505529.

Dolata, U. (2020). Plattform-Regulierung. Koordination von Märkten und Kuratierung von Sozialität im Internet. Berliner Journal für Soziologie, 29(3-4), 179-206. 
Dörre, K. (2017). Digitalisierung - neue Prosperität oder gesellschaftliche Spaltungen. In H. Hirsch-Kreinsen, P. Ittermann \& J. Niehaus (Hrsg.), Digitalisierung industrieller Arbeit. Die Vision Industrie 4.0 und ibre sozialen Herausforderungen (S. 365-382). Baden-Baden: Nomos.

Dörre, K., \& Haubner, T. (2012). Landnahme durch Bewährungsproben - Ein Konzept für die Arbeitssoziologie. In K. Dörre, D. Sauer \& V. Wittke (Hrsg.), Kapitalismustheorie und Arbeit. Neue Ansätze soziologischer Kritik (S.63-106). Frankfurt/New York: Campus.

Ellguth, P., \& Kohaut, S. (2020). Tarifbindung und betriebliche Interessenvertretung. Aktuelle Ergebnisse aus dem Betriebspanel 2019. WSI-Miteilungen, 73(4), 278-285.

Fligstein, N. (1990). The Transformation of Corporate Control. Cambridge: Havard University Press.

Frey, C.B., \& Osborne, M.A. (2013). The future of employment: How suscetible are jobs to computerisation? Working Paper. Oxford: University of Oxford.

Froud, J., Johal, S., Leaver, A., \& Williams, K. (2006). Financialization and Strategy. Narrative and Numbers. London/New York: Routledge.

Gereffi, G., Humphrey, J., \& Sturgeon, T. (2005). The Governance of Global Value Chains. Review of International Political Economy, 12(1), 78-104.

Gerlmaier, A., \& Geiger, L. (2018), Produktionsarbeit in Zeiten von Industrie 4.0: Was wissen Unternehmen und Beschäftigte über eine gesundheitsgerechte Gestaltung von Arbeit? IAQ-Report 2018-02. Duisburg: Institut Arbeit und Qualifikation.

Gerst, D. (2016). Roboter erobern die Arbeitswelt. In L. Schröder \& H.-J. Urban (Hrsg.), Gute Arbeit. Digitale Arbeitswelt - Trends und Anforderungen (S. 279-293). Frankfurt am Main: Bund-Verlag.

Glissmann, W., \& Peters, K. (2000). Mehr Druck durch mehr Freiheit. Die neue Autonomie in der Arbeit und ihre paradoxen Folgen, Hamburg: VSA.

Haipeter, T. (2016). Finanzmarktkapitalismus und Arbeit. In T. Haipeter, E. Latniak \& S. Lehndorff (Hrsg.), Arbeit und Arbeitsregulierung im Finanzmarktkapitalismus (S. 11-43). Wiesbaden: Springer.

Haipeter, T., Hertwig, M., \& Rosenbohm, S. (2019). Employee Representation in Multinational Companies. The Articulation of Interests in Multilevel Action Fields. Cham: Palgrave Macmillan.

Haipeter, T., Korflür, I., \& Schilling, G. (2018). Neue Koordinaten für eine proaktive Betriebspolitik. Erfahrungen aus dem Gewerkschaftsprojekt „Arbeit 2020 in NRW“. WSI-Mitteilungen, 71(3), 219-226.

Haipeter, T., Bromberg, T., \& Slomka, C. (2016). Angestellte als Machtquelle. Neue Initiativen der Interessenvertretung von Industrieangestellten im Betrieb. Wiesbaden: Springer VS.

Haipeter, T, Latniak, E., \& Lehndorff, S. (Hrsg.) (2016). Arbeit und Arbeitsregulierung im Finanzmarktkapitalismus: Chancen und Grenzen eines soziologischen Analysekonzepts. Wiesbaden: Springer VS. 
Hirsch-Kreinsen, H. (2018a). Einleitung: Digitalisierung industrieller Arbeit. In H. Hirsch-Kreinsen, P. Ittermann \& J. Niehaus (Hrsg.), Digitalisierung industrieller Arbeit: Die Vision Industrie 4.0 und ihre sozialen Herausforderungen (2. Auflage, S. 13-32). Baden-Baden: Nomos/edition sigma.

Hirsch-Kreinsen, H. (2018b). Arbeit 4.0: Pfadabhängigkeit statt Disruption. Soziologisches Arbeitspapier Nr. 52. Dortmund: TU Dortmund.

Hirsch-Kreinsen, H. (2018c). Multinationale Unternehmen. In F. Böhle, F. Voß, G. Günter \& G. Wachtler (Hrsg.), Handbuch Arbeitssoziologie, Bd. 2.: Akteure und Institutionen (2. Auflage, S. 277-298). Wiesbaden: Springer VS.

Hoose, F. (2018). Digitale Arbeit. Strukturen eines Forschungsfeldes. IAQ-Forschung 2018/03. Duisburg: Institut Arbeit und Qualifikation.

Howaldt, J., Kopp, R., \& Schultze, J. (2018). Zurück in die die Zukunft? Ein kritischer Blick auf die Diskussion zur Industrie 4.0. In H. Hirsch-Kreinsen, P. Ittermann, \& J. Niehaus (Hrsg.), Digitalisierung industrieller Arbeit. Die Vision Industrie 4.0 und ibre sozialen Herausforderungen (2. Auflage, S. 347-364). Baden-Baden: Nomos.

Huws, U. (2016). Logged Labour. A New Paradigm of Work Organisation? Work Organisation, Labour \& Globalisation, 10(1), 7-26.

Huws, U., Spencer, N. H., \& Joyce, S. (2016). Crowd work in Europe. Preliminary results from a survey in the UK, Sweden, Germany, Austria and the Netherlands. FEPS Studies, December 2016. Brüssel: Foundation for European Progressive Studies (FEPS).

Ittermann, P., \& Niehaus, J. (2018). Industrie 4.0 und Wandel von Industrie revisited.: Forschungsstand und Trendbestimmungen. In H. Hirsch-Kreinsen, P. Ittermann \& J. Niehaus (Hrsg.), Digitalisierung industrieller Arbeit: Die Vision Industrie 4.0 und ibre sozialen Herausforderungen (2. Auflage, S.33-60). Baden-Baden: Nomos/edition sigma.

Kädtler, J. (2009). Finanzialisierung und Finanzmarktrationalität. Zur Bedeutung konventioneller Handlungsorientierungen im gegenwärtigen Kapitalismus. SOFI-Arbeitspapier 5. Göttingen: SOFI.

Kagermann, H. (2014). Chancen von Industrie 4.0 nutzen. In T. Bauernhansl, M. ten Hompel \& B. Vogel-Heuser (Hrsg.), Industrie 4.0 in Produktion, Automatisierung und Logistik. Anwendung, Technologien, Migration (S. 603-614). Wiesbaden: Springer.

Kalkowski, P., \& Mickler, O. (2009). Antinomien des Projektmanagements. Eine Arbeitsform zwischen Direktive und Freiraum. Berlin: edition sigma.

Kern, H., \& Schumann, M. (1984). Das Ende der Arbeitsteilung. Rationalisierung in der industriellen Produktion. München: Beck.

Kondratjew, N. D. (1926). Die langen Wellen der Konjunktur. Archiv für Sozialwissenschaft und Sozialpolitik 56, 573-609.

Kuhlmann, M. (2018). Montagearbeit 4.0? Eine Fallstudie zu Arbeitswirkungen und Gestaltungsperspektiven digitaler Werkerführung. WSI-Mitteilungen, 71(3), 182-188. 
Latniak, E., \& Gerlmaier, A. (2019). Ende der Arbeitsgestaltung durch Digitalisierung? In A. Gerlmaier \& E. Latniak (Hrsg.), Handbuch psycho-soziale Gestaltung digitaler Produktionsarbeit (S. 19-36). Wiesbaden: Springer.

Lazonick, W., \& O'Sullivan, M. (2000). Maximising Shareholder Value: An Ideology of Corporate Governance. Economy and Society, 29(1), 13-35.

Lehndorff, S. (2006). Einleitung: das Politische in der Arbeitspolitik. In S. Lehndorff (Hrsg.), Das Politische in der Arbeitspolitik: Ansatzpunkte für eine nachhaltige Arbeits- und Arbeitszeitgestaltung (S. 7-29). Berlin: edition sigma.

Leimeister, J. M., Durward, D., \& Zogaj, S. (2016). Crowd Worker in Deutscland. Eine empirische Studie zum Arbeitsumfeld auf externen Crowdsourcing-Plattformen. Hans-Böckler-Stiftung Study Nr. 323. Düsseldorf: Hans-Böckler-Stiftung.

Lerch, C., Jäger, A., \& Spomenca, M. (2017). Wie digital ist Deutschlands Industrie wirklich? Mitteilungen aus der ISI-Erhebung Modernisierung der Produktion Nr. 71. Karslruhe: Fraunhofer ISI.

Lévesque, C., \& Murray, G. (2013). Gewerkschaftsmacht verstehen: Ressourcen und Fähigkeiten zur Erneuerung strategischen Handlungsvermögens. In S. Schmalz \& K. Dörre (Hrsg.), Comeback der Gewerkschaften? Machtressourcen, innovative Praktiken, internationale Perspektiven (S.39-55). Frankfurt/New York: Campus.

Lipietz, A. (1985). Akkumulation, Krisen und Auswege aus der Krise. Einige methodische Überlegungen zum Begriff der Regulation, Prokla, 58, 109-138.

Lipietz, A. (1997). The Post-Fordist World: Labour Relations, International Hierarchy and Global Ecology. Review of International Political Economy, 4(1), 1-41.

Maasen, S., \& Passoth, J.-H. (Hrsg.) (2020). Soziologie des Digitalen - Digitale Soziologie? Soziale Welt. Sonderband 23. Baden-Baden: Nomos.

Marres, N. (2017). Digital Sociology: The Reinvention of Social Research. Cambridge: Polity.

Mason, P. (2015). Postcapitalism. A Guide to Our Future. London: Penguin Random House.

Meyer, U. (2020). Industrie 4.0 als sozio-technische Zukunftsvorstellung. Zur Bedeutung von organisationaler Sinnerzeugung und stiftung. In S. Maasen \& J.-H. Passoth (Hrsg.), Soziologie des Digitalen - Digitale Soziologie? Soziale Welt. Sonderband 23 (S. 349-377). Baden-Baden: Nomos.

Minssen, H. (2017). Industrie 4.0: Ein Strukturbruch? In F. Hoose, F. Beckmann \& A.-L. Schönauer (Hrsg.), Fortsetzung folgt: Kontinuität und Wandel von Wirtschaft und Gesellschaft (S. 117-135). Wiesbaden: Springer VS.

Niehaus, J. (2017). Mobile Assistenzsysteme für Industrie 4.0: Gestaltungsoptionen zwischen Autonomie und Kontrolle. FGW-Studie Digitalisierung von Arbeit. Düsseldorf: FGW.

OECD (2019). The Future of Work. OECD Employment Outlook 2019. Paris: OECD.

Parker, S. K., \& Grote, G. (2019). Automation, Algorithms, and beyond: Why Work Design Matters More Than Everything in a Digital World. Applied Psychology. Online first: https://doi.org/10.1111/apps.12241. 
Pfeiffer, S. (2015). Warum reden wir eigentlich über Industrie 4.0? Auf dem Weg zum digitalen Despotismus. Mittelweg 36, 24(6), 14-36.

Pfeiffer, S. (2018). Technisierung von Arbeit. In F. Böhle, G. G. Voß \& G. Wachtler (Hrsg.), Handbuch Arbeitssoziologie, Bd. 1: Arbeit, Strukturen und Prozesse (2. Auflage, S. 321-357). Wiesbaden: Springer VS.

Piore, M. J., \& Sabel, C. (1985). Das Ende der Massenproduktion. Berlin.

Rifkin, J. (2014). The Zero Marginal Cost Society. The Internet of Things, the Collaborative of the Commons and the Eclipse of Capitalism. New York: Palgave Macmillan.

Rothe, I., Wischniewski, S., Tegtmeier, P., \& Tisch, A. (2019). Arbeiten in der digitalen Transformation - Chancen und Risiken für die menschengerechte Arbeitsgestaltung. Zeitschrift für Arbeitswissenschaft, 73(3), 246-251.

Schiller, D. (1999). Digital Capitalism. Networking the Global Market System. Cambridge: MIT Press.

Schmidt, F. A. (2016). Arbeitsmärkte in der Plattformökonomie. Zur Funktionsweise und den Herausforderungen von Crowdwork und Gigwork. Berlin: Friedrich-EbertStiftung.

Schramm, F., \& Tietgen-Simonsen, M. (2019). Crowdworking - Menetekel und empirische Evidenz. In H. Hanau \& W. Matiaske (Hrsg.), Entgrenzung von Arbeitsverhältnissen: Arbeitsrechtliche und sozialwissenschaftliche Perspektiven (S. 1124). Baden-Baden: Nomos/edition sigma.

Schumpeter, J. A. (1961). Konjunkturzyklen. Eine theoretische, historische und statistische Analyse des kapitalistischen Prozesses. Göttingen: Vandenhoeck \& Ruprecht.

Schwemmle, M., \& Wedde, P. (2012). Digitale Arbeit in Deutschland: Potenziale und Problemlagen. Bonn: Friedrich-Ebert-Stiftung. Verfügbar unter: http://library .fes.de/pdf-files/akademie/09324.pdf [20.11.2020].

Schwemmle, M., \& Wedde, P. (2018). Alles unter Kontrolle? Arbeitspolitik und Arbeitsrecht in digitalen Zeiten. Bonn: Friedrich-Ebert-Stiftung. Verfügbar unter: http://library.fes.de/pdf-files/wiso/14087.pdf [19.11.20].

Spath, D. (Hrsg.) (2013). Produktionsarbeit der Zukunft - Industrie 4.0. Stuttgart: Fraunhofer Verlag.

Staab, P., \& Butollo, F. (2018). Digitaler Kapitalismus - Wie China das Silicon Valley herausfordert. WISO direkt, 03/2018. Bonn: Friedrich-Ebert-Stiftung. Verfügbar unter: http://library.fes.de/pdf-files/wiso/14037.pdf [15.01.2018].

Staab, P., \& Nachtwey, O. (2016). Die Digitalisierung der Dienstleistungsarbeit. АриZ, 66(18-19), 24-31.

Staab, P., \& Prediger, L. J. (2019). Digitalisierung und Polarisierung. Eine Literaturstudie zu den Auswirkungen des digitalen Wandels auf Sozialstruktur und Betriebe. FGW-Studie. Digitalisierung von Arbeit 19. Düsseldorf: FGW.

Sträter, O. (2019). Wandel der Arbeitsgestaltung durch Digitalisierung. Zeitschrift für Arbeitswissenschaft, 73(3), 252-260.

Streeck, W. (1998). Industrielle Beziehungen in einer internationalisierten Wirtschaft. In U. Beck (Hrsg.), Politik der Globalisierung (S. 169-202). Frankfurt: Suhrkamp. 
Südekum, J. (2018). Digitalisierung und die Zukunft der Arbeit: Was ist am Arbeitsmarkt passiert und wie soll die Wirtschaftspolitik reagieren? IZA Standpunkte, 90. Bonn: IZA.

Sundararajan, A. (2016). The Sharing Economy. The End of Employment and the Rise of Crowd-Based-Capitalism. Cambridge: MIT Press.

Tullius, K. (2020). Digitalisierung und Systemische Rationalisierung im Finanzdienstleistungssektor - Folgen für Angestelltenarbeit an der „Front-Line“. SOFI Arbeitspapier/ SOFI Working Paper, 2020-18. Göttingen: SOFI.

Urban, H.-J. (2016). Arbeiten in der Wirtschaft 4.0. Über kapitalistische Rationalisierung und digitale Humanisierung. In L. Schröder \& H.-J. Urban (Hrsg.), Gute Arbeit. Digitale Arbeitswelt - Trends und Anforderungen (S. 21-45). Frankfurt am Main: Bund-Verlag.

Urzì Brancati, M.C., Pesole, A., \& Férnandéz-Macías, E. (2020). New evidence on platform workers in Europe. JRC Science for Policy Report. Luxemburg: Publications Office of the European Union.

Windolf, P. (2008). Eigentümer ohne Risiko. Die Dienstklasse des Finanzmarkt-Kapitalismus. Zeitschrift für Soziologie, 37(6), 516-535.

Zika, G., Helmrich, R., Maier, T., Weber, E., \& Wolter, M. I., (2018). Arbeitsmarkteffekte der Digitalisierung bis 2035. Regionale Branchenstruktur spielt eine wichtige Rolle. IAB-Kurzbericht 09/2018. Nürnberg: IAB. 